\title{
FINITE ELEMENT SOLUTION FOR ADVECTION AND NATURAL CONVECTION FLOWS
}

\author{
B. Ramaswamy \\ Department of Mechanical Engineering and Applied Mechanics, The University of Michigan, Ann Arbor, \\ MI 48109, U.S.A.
}

(Received 10 March 1987; in revised form 28 October 1987)

\begin{abstract}
A new equal order velocity-pressure finite element procedure is presented for the calculation of 2-D viscous, incompressible flows of a recirculating nature. As in the finite difference procedures, velocity and pressure are uncoupled and the equations are solved one after the other. In this splitting-up method, an auxilary velocity field is computed first, which accounts for all contributions to the acceleration, except pressure, and satisfies the velocity boundary conditions. Then, the final velocities are evaluated by adding to the auxilary velocities pressure contributions which are computed to satisfy the continuity equation. The effectiveness is illustrated via example problems of $2-\mathrm{D}$ advection and natural convection flows.
\end{abstract}

\section{INTRODUCTION}

This paper presents the development of a 2-D code for solving the viscous, incompressible Navier-Stokes (NS) equations. The purpose of this research is to identify a suitable approach to finite element analysis of recirculating flows involving heat and mass transfer. The desire to follow a physically meaningful approach and to specify the boundary conditions easily, made to consider the so-called primitive variables-i.e. velocity-pressure formulation.

The major emphasis during the present investigation has been on the development of both computational and modelling techniques that contribute to the efficiency of the finite element method as applied to fluid mechanics and heat transfer problems. The sequential approach is commonly used in the finite difference method (FDM) [1-3]. However, considerably different numerical techniques have to be employed here to allow finite element discretization, from the user's point of view, to retain all the flexibility and ease of the finite element method (FEM) in dealing with complex domains and boundary conditions.

The transient flow of incompressible viscous fluid is characterized by the hyperbolic, parabolic and elliptic properties. The hyperbolic and parabolic properties result from the convective and diffusive terms of the NS equations, respectively and elliptic property is due to the incompressibility of fluid. Therefore, it is very natural to decompose the time integration of the incompressible NS equations into three separated steps. In the present scheme, the hyperbolic convective equation is temporally integrated in the explicit Euler manner to save the computational efforts. Since the application of the explicit Euler scheme induces negative numerical viscosity and leads to non-linear instability, the balancing tensor viscosity is added into the physical viscosity. The fully implicit (FI) time integration is used for the incompressibility equation, because the fluid is always incompressible. The same time integration scheme is applied to the time integration of the pressure gradient term, since pressure plays a role of the Lagrange multiplier for the incompressibility constraint from the view point of variational principle. Such time integration yields the elliptic pressure Poisson equation, which is solved by the skyline version of the Gauss-elimination technique to save the computational storage demand in the present computation.

The performance of the above mentioned method as applied to 6 example problems will also be indicated. The 6 problems are those of wall driven cavity flow, sudden expansion problem, flow over a square step, flow through a flat plate, flow around a stationary sphere and natural convection heat transfer problems. Where possible the results are compared with existing solutions obtained by other numerical methods. A satisfactory correlation exists where such comparisons can be made. The results complement and extend those obtained during previous theoretical and numerical investigation. 


\section{BASIC EQUATIONS}

The equations to be solved are derived from the basic physical principles of conservation of mass, momentum and energy. An unsteady flow Eulerian formulation will be employed. The equations are expressed in cartesian tensor notation with summation over repeated indices implied.

Application of the principles of mass conservation results in the continuity equation:

$$
\left(\rho u_{i}\right)_{, i}=0,
$$

where $\rho$ denotes the mass density, and $u_{i}$ the components of the velocity vector in the $x_{i}$-coordinate direction.

From a balance of linear momentum surface and body forces on an arbitrarily small fluid volume, the Cauchy equation can be obtained as:

$$
\frac{\partial u_{i}}{\partial t}+\rho u_{j} u_{i, j}=\sigma_{i j, j}+\rho f_{i},
$$

where $f_{i}$ denotes the components of the body force vector per unit mass and $\sigma_{i j}$ the stress tensor. For a Newtonian fluid the constitutive relation for the stress tensor in terms of the mean stress $-p$ and rate of strain tensor $u_{i, j}$ is:

$$
\sigma_{i j}=-p \delta_{i j}+2 \mu\left(u_{i, j}+u_{j, i}\right)-\frac{2}{3} \mu\left(u_{k, k}\right) \delta_{i j}
$$

where $\mu$ is the coefficient of viscosity.

The energy equation may be expressed in terms of the thermodynamic property of enthalpy, $h$, as:

$$
\frac{\partial h}{\partial t}+\rho u_{i} h_{, i}=u_{i} p_{, i}+u_{i, j}\left(\sigma_{i j}+p \delta_{i j}\right)+\left(K T_{, i}\right)_{, i}
$$

where Fourier's constitutive relation for the heat flux vector in terms of the temperature gradient in isotropic media has been employed, $K$ being the coefficient of thermal conductivity and the mean stress has been set equal to the negative of the thermodynamic pressure.

The following simplifying assumptions are made:

1. The boussinesq approximation that density variation is negligible except in the body force term $f_{i}$ where temperature induced variations give rise to a body force which contributes to fluid motion.

2. The density satisfies an equation of state of the form $\rho=\rho_{0}\left[1+\beta\left(T-T_{0}\right)\right]$ where the subscript 0 denotes a reference state and $\beta$ is the coefficient of thermal expansion.

3. The enthalpy is a function of temperature alone with $d h=C_{\mathrm{P}} d T$, where $C_{P}$ is the specific heat at constant pressure.

4. The pressure gradient is sufficiently small that the term $u_{i} p_{. i}$ may be neglected in eqn (4).

5. Constant physical properties, $\mu, C_{\mathrm{P}}, \beta$ and $K$.

6. The body force vector per unit mass $f_{i}$ is constant.

With these assumptions the system of equations to be solved may be expressed in terms of the dependent variables $u_{i}, p^{\prime}$ and $\theta$ as follows:

$$
\begin{aligned}
u_{i, i} & =0 \\
\rho_{0} \frac{\partial u_{i}}{\partial t}+\rho_{0} u_{j} u_{i, j} & =-p_{, i}^{\prime}+2 \mu\left(u_{i, j}+u_{j, i}\right)_{, j}+\rho_{0} \beta \theta f_{i} \\
\frac{\partial \theta}{\partial t}+\rho_{0} C_{\mathrm{p}} u_{j} \theta_{, j} & =K \theta_{, j j}+\mu \phi
\end{aligned}
$$

where

$$
\begin{aligned}
p^{\prime} & =p-\rho_{0} f_{i} x_{i} \\
\theta & =T-T_{0} \\
\phi & =2 u_{i, j}\left(u_{i, j}+u_{j, i}\right)
\end{aligned}
$$


Equation (5) may be regarded as imposing a constraint on the vector field $u_{i}$. The vector field of velocity must be divergence free or solenoidal. These equations are applicable to low speed flow of gases as well as the flow of liquids which are essentially incompressible. Assumption (4) in effect models the energy addition to an elemental fluid element as a constant pressure process. Implicit allowance is made for the compressibility of a gas as required by thermodynamic considerations, through the use of the enthalpy function $h=e+p / \rho$ rather than the internal energy $e$ as the energy variable.

It is convenient to express the above equations in non-dimensional form. Introducing reference quantities $u^{*}, \theta^{*}$ and $L$, the velocity components, temperature difference and coordinates may be normalized. The pressure will be normalized with respect to $\rho_{0}\left(u^{*}\right)^{2}$. Employing upper case characters to denote the normalized variables, the resulting equations are:

$$
\begin{aligned}
U_{i, i} & =0 \\
\frac{\partial U_{i}}{\partial t}+U_{j} U_{i, j} & =-P_{, i}+\left(\frac{v}{u^{*} L}\right)\left(U_{i, j}+U_{j, i}\right)_{, j}+\left(\frac{\beta \theta^{*} g L}{\left(u^{*}\right)^{2}}\right) \Theta \hat{f}_{i} \\
\frac{\partial \Theta}{\partial t}+U_{j} \Theta_{, j} & =\left(\frac{\alpha}{L u^{*}}\right) \Theta_{. j j}+\left(\frac{v}{u^{*} L}\right)\left(\frac{\left(u^{*}\right)^{2}}{C_{\mathrm{p}} \theta^{*}}\right) \phi
\end{aligned}
$$

where $\alpha=K /\left(\rho_{0} C_{\mathrm{P}}\right)$ is the thermal diffusivity, $v=\mu / \rho_{0}$ the kinematic viscosity and $f_{i}=g \hat{f}_{i}$ with $\hat{f}_{i}$ the components of a unit vector and $g$ the gravitational constant. The non-dimensional coefficients in these equations may be expressed in terms of traditional dimensionless group such as:

-Reynolds number,

$$
\operatorname{Re}=\frac{u^{*} L}{v}
$$

-Prandtl number,

$$
\operatorname{Pr}=\frac{v}{\alpha}
$$

- Grashof number,

$$
\mathrm{Gr}=\frac{\beta g \theta^{*} L^{3}}{v^{2}}
$$

-Eckert number,

$$
\mathrm{Ec}=\frac{\left(u^{*}\right)^{2}}{C_{\mathrm{P}} \theta^{*}}
$$

-Peclet number,

$$
\operatorname{Pe}=\operatorname{Pr} \cdot \operatorname{Re}=\frac{u^{*} L}{\alpha}
$$

-Rayleigh number,

$$
\mathrm{Ra}=\mathrm{Gr} \cdot \operatorname{Pr}=\frac{\beta g \theta^{*} L^{3}}{v \alpha}
$$

The particular groups employed and resultant form of the equations are determined in part by the choice for the reference velocity $u^{*}$. In forced convection problems, a characteristic velocity $u^{*}=u_{\infty}$ may be identified and the coefficients are usually expressed in terms of the group Re, Gr, $\mathrm{Pe}$ and Ec. In the case of natural convection where a characteristic velocity may not be identified a priori $u^{*}=\alpha / L$ or $v / L$ is typically chosen with the coefficients being expressed in terms of $\operatorname{Pr}$ and $\mathrm{Ra}$ with the internal viscous dissipation neglected. In this paper the theoretical aspects is explained only for natural convection but in the illustrations both advection and natural convection problems were discussed. If the internal heating by viscous dissipation is neglected, $\alpha / L$ chosen as $u^{*}$ and the Rayleigh and Prandtl numbers employed as dimensionless groups, eqns (12) and (13) may be rewritten as: 


$$
\begin{aligned}
& \frac{\partial U_{i}}{\partial t}+U_{j} U_{i, j}=-P_{, j}+\operatorname{Pr}\left[\left(U_{i, j}+U_{j . i}\right)_{, j}+\operatorname{Ra} \hat{f}_{i} \Theta\right] \\
& \frac{\partial \Theta}{\partial t}+U_{j} \Theta_{, j}=\Theta_{, j}
\end{aligned}
$$

The transient flow of incompressible viscous fluid is characterized by the hyperbolic, parabolic and elliptic properties. Therefore, eqn (14) can be decomposed into the following three fractional steps.

-advection:

$$
\begin{aligned}
& \frac{\partial U_{i}}{\partial t}+U_{j} U_{i, j}=0 \\
& \frac{\partial \Theta}{\partial t}+U_{j} \Theta_{, j}=0
\end{aligned}
$$

-diffusion:

$$
\begin{gathered}
\frac{\partial U_{i}}{\partial t}-\operatorname{Pr}\left(U_{i, j}+U_{j, i}\right)_{, j}=\operatorname{PrRa} \hat{f}_{i} \Theta \\
\frac{\partial \Theta}{\partial t}-\Theta_{. j j}=0
\end{gathered}
$$

-continuity:

$$
\begin{aligned}
\frac{\partial U_{i}}{\partial t}+P_{, i} & =0 \\
U_{i, i} & =0 .
\end{aligned}
$$

The above three steps have different properties from the view point of the classification of partial differential equations. The convection step has the hyperbolic property and is integrated in the explicit Euler method. However, the explicit Euler method is the first-order in accuracy and unconditionally unstable owing to the introduction of negative numerical diffusivity. In order to compensate for the negative diffusivity, the same amount of balancing tensor viscosity is added artificially into the physical diffusivity as follows.

$$
\begin{aligned}
& \frac{\partial U_{i}}{\partial t}+U_{j} U_{i, j}=-P_{. i}+\left(\operatorname{Pr}+\frac{\Delta t}{2} U_{i} U_{j}\right)\left(U_{i, j}+U_{i, i}\right)_{, j}+\operatorname{PrRa} \hat{f}_{i} \Theta \\
& \frac{\partial \Theta}{\partial t}+U_{j} \Theta_{, j}=\left(1+\frac{\Delta t}{2} U_{i} U_{j}\right) \Theta_{, j j}
\end{aligned}
$$

where $\Delta t$ is the time increment.

To complete the formulation of the basic equations a set of boundary and initial conditions are required. For the hydrodynamic part of the problem, the continuum boundary conditions are of two types; those specifying velocity and those specifying stress. Application of the specified velocity condition in discrete form is straightforward. The equation for the particular velocity component at a boundary node is replaced by a constraint condition enforcing the proper boundary value. This in general can be defined as:

$$
U_{i}=\hat{U}_{i}, \quad \text { on } S_{1}
$$

For boundary conditions specified in terms of stress components, the surface force is supposed to be given on boundary $S_{2}$ i.e.:

$$
t_{i}=\Sigma_{i j} \cdot n_{j}=\hat{t}_{i}, \quad \text { on } S_{2}
$$

where $\Sigma_{i j}$ denotes the non-dimensional stress tensor $\left(\sigma_{i j}\right) /\left[\rho_{0}\left(u^{*}\right)^{2}\right]$. 
The thermal part of the problem requires a temperature or heat flux to be specified on all parts of the boundary. Symbolically, these conditions are expressed by:

$$
\begin{aligned}
\Theta & =\hat{\Theta}, \quad \text { on } S_{3} \\
q & =\Theta_{, i} \cdot n_{i}=\hat{q}, \quad \text { on } S_{4}
\end{aligned}
$$

for the heat transfer region. In eqns (24)-(27) $n_{i}$ is the direction cosine of the outward normal on the boundary with respect to $X_{j}$-axis. Moreover, the subsets $S_{1}, S_{2}, S_{3}$ and $S_{4}$ of $S$ which satisfy the following conditions:

$$
\begin{aligned}
& \overline{S_{1} \cup S_{2}}=S \\
& S_{1} \cap S_{2}=\emptyset
\end{aligned}
$$

and

$$
\begin{aligned}
& \overline{S_{3} \cup S_{4}}=S \\
& S_{3} \cap S_{4}=\emptyset
\end{aligned}
$$

The superposed bar in eqns (28) and (30), represents the total boundary enclosing the fluid and the energy transfer region, respectively and $\emptyset$ in eqns (29) and (31) denotes the empty set.

The initial conditions for convective-conductive heat transfer problems consists of specifying the value of velocity and temperature at the initial time:

$$
\begin{aligned}
& U_{i}\left(X_{i}, 0\right)=U_{i}^{(0)}\left(X_{i}\right), \\
& \Theta\left(X_{i}, 0\right)=\Theta^{(0)}\left(X_{i}\right),
\end{aligned}
$$

with the initial velocity $U_{i}^{(0)}\left(X_{i}\right)$, satisfying the incompressibility condition,

$$
U_{i, i}^{(0)}=0
$$

and

$$
U_{i}\left(X_{i}, 0\right) \cdot n_{i}=\hat{U}_{i}\left(X_{i}, 0\right) \cdot n_{i}, \quad \text { on } S_{1}
$$

\section{VELOCITY CORRECTION METHOD}

To obtain the velocity, pressure and temperature at time $t^{n+1}$, the velocity correction method is successfully used in the present analysis. At first, an intermediate velocity fields $\tilde{U}_{i}^{n+1}$ not satisfying the incompressibility constraint, is derived from a time-discretized version of the momentum equation in which the pressure terms are omitted. Then, the field $\tilde{U}_{i}^{n+1}$ is decomposed into the sum of a vector field with zero divergence and a vector field with zero curl. The divergenceless component is the end-of-step velocity vectors $U_{i}^{n+1}$, whereas the irrotational one is related to the gradient of the pressure field $P^{n+1}$.

The intermediate nodal velocity vector fields $\tilde{U}_{i}^{n+1}$, not satisfying the incompressibility condition is derived resulting from the previous cycle's velocity vectors, pressure, body forces and temperature by employing a purely explicit Euler's first-order scheme:

$$
\begin{aligned}
& \tilde{U}_{i}^{n+1}=U_{i}^{n}-\Delta t\left\{U_{j}^{n} U_{i, j}^{n}-\left(\operatorname{Pr}+\frac{\Delta t}{2} U_{i}^{n} U_{j}^{n}\right)\left(U_{i, j}^{n}+U_{j, i}^{n}\right)_{, j}-\operatorname{PrRa} \Theta^{n} \hat{f}_{i}^{n}\right\} \\
& \tilde{U}_{i}^{n+1}=\hat{U}_{i}, \quad \text { on } S_{1} .
\end{aligned}
$$

Once the intermediate velocity has been computed, the end-of-step velocity $U_{i}^{n+1}$ is obtained by adding to $\tilde{U}_{i}^{n+1}$ the dynamical effect of the still unknown pressure $P^{n+1}$ which is to be determined so that the incompressibility condition remains satisfied.

$$
U_{i}^{n+1}=\tilde{U}_{i}^{n+1}-\Delta t P_{, i}^{n+1}
$$


Taking divergence on both sides of eqn (38) together with incompressibility constraint $U_{i, i}^{n+1}=0$, the following equation for pressure can be derived.

$$
P_{, i i}^{n+1}=\frac{1}{\Delta t} \tilde{U}_{i . j}^{n+1} .
$$

To solve the above eqn (39) by the finite element method, the following boundary conditions are applied.

$$
\begin{aligned}
P & =\hat{P}, \quad \text { on } S_{1} \\
r & =P_{. i}^{n+1} \cdot n_{i}=\hat{r}, \quad \text { on } S_{2}
\end{aligned}
$$

Once the pressure has been determined from eqn (39), nodal velocities can be calculated from eqn (38). The new velocities given by eqn (38) clearly satisfies the weak form of the incompressibility constraint, as well as the boundary conditions. Then, the temperature at all nodal points can be calculated by employing a purely explicit Euler's scheme to the transport eqn (23).

$$
\begin{aligned}
& \Theta^{n+1}=\Theta^{n}-\Delta t\left\{U_{j}^{n+1} \Theta_{, j}^{n}+\left(1+\frac{\Delta t}{2}\right)\left(U_{i}^{n+1} U_{j}^{n+1}\right) \Theta_{. j j}^{n}\right\} \\
& \Theta^{n+1}=\hat{\Theta}, \text { on } S_{1}
\end{aligned}
$$

This completes the updating of all quantities.

\section{FINITE ELEMENT METHOD}

Based on the velocity $U_{i}^{n}$, pressure $P^{n}$ and temperature $\Theta^{n}$ computed at the previous time point, the velocity, pressure and temperature for the next time step can be calculated. Equations (36), (38), (39) and (42) were discretized by the standard Galerkin finite element method. Assume that the flow field is divided into a number of small domains called finite elements. For velocity, pressure and temperature, the interpolation equations can be introduced as follows:

$$
\begin{aligned}
& U_{i}=\phi_{x} U_{x i} \\
& P=\phi_{x} P_{x} \\
& \Theta=\phi_{x} \Theta_{x}
\end{aligned}
$$

where $\phi_{\alpha}$ is the interpolation function, $U_{\alpha i}$ denotes the nodal value of velocity at the $\alpha$ th node of the finite element in the $i$ th direction, $P_{\alpha}$ means the nodal value of pressure at the $\alpha$ th node, and $\Theta_{\alpha}$ is the nodal value of temperature at the $\alpha$ th node. The application of the finite element procedure to equations (36), (38), (39) and (42) leads to the following set of equations:

$$
\begin{aligned}
& \bar{M}_{\alpha \beta} \tilde{U}_{\beta i}^{n+1}=\bar{M}_{\alpha \beta} U_{\beta i}^{n}-\Delta t\left(K_{\alpha \beta \gamma j} U_{\beta j}^{n} U_{\gamma i}^{n}+S_{\alpha i \beta j} U_{\beta j}^{n}+S_{\alpha i \beta j}^{\prime} U_{\beta j}^{n}-N_{\alpha} \Theta_{\alpha}^{n} \hat{f}_{\alpha i}^{n}-\hat{\Omega}_{\alpha i}^{n}\right) \\
& \bar{M}_{\alpha \beta} U_{\beta i}^{n+1}=\bar{M}_{\alpha \beta} \tilde{U}_{\beta i}^{n+1}-\Delta t H_{\alpha i \beta} P_{\beta}^{n+1} \\
& A_{\alpha \beta} P_{\beta}^{n+1}=-\frac{1}{\Delta t} H_{\alpha i \beta} \tilde{U}_{\beta i}^{n+1}+\hat{\Sigma}_{\alpha} \\
& \bar{M}_{\alpha \beta} \Theta_{\beta}^{n+1}=\bar{M}_{\alpha \beta} \Theta_{\beta}^{n}-\Delta t\left(K_{\alpha \beta \gamma j} U_{\beta j}^{n+1} \Theta_{\gamma}^{n}+A_{\alpha \beta} \Theta_{\beta}^{n}+A_{\alpha \beta}^{\prime} \Theta_{\beta}^{n}-\hat{\Sigma}_{1 \alpha}\right)
\end{aligned}
$$

where

$$
\begin{aligned}
M_{\alpha \beta} & =\int_{V}\left(\phi_{\alpha} \phi_{\beta}\right) \mathrm{d} V, \\
H_{\alpha i \beta} & =\int_{V}\left(\phi_{\alpha, i} \phi_{\beta}\right) \mathrm{d} V, \\
K_{\alpha \beta ; j} & =\int_{V}\left(\phi_{\alpha} \phi_{\beta, j} \phi_{\gamma}\right) \mathrm{d} V,
\end{aligned}
$$




$$
\begin{aligned}
A_{\alpha \beta} & =\int_{V}\left(\phi_{\alpha, i} \phi_{\beta, j}\right) \mathrm{d} V, \\
N_{\alpha} & =\operatorname{RaPr} \int_{V}\left(\phi_{\alpha}\right) \mathrm{d} V, \\
\hat{\Omega}_{\alpha i} & =\operatorname{Pr} \int_{S}\left\{\phi_{\alpha}\left(u_{i, j}^{n}+u_{j, i}^{n}\right) \cdot n_{j}\right\} \mathrm{d} S, \\
\hat{\Sigma}_{\alpha} & =\int_{S}\left(\phi_{\alpha} p_{, i}^{n+1} \cdot n_{i}\right) \mathrm{d} S, \\
\hat{\Sigma}_{1 \alpha} & =\int_{S}\left(\phi_{\alpha} p_{, i}^{n+1} \cdot n_{i}\right) \mathrm{d} S, \\
S_{\alpha i \beta j} & =\operatorname{Pr} \int_{V}\left(\phi_{\alpha, k} \phi_{\beta, k}\right) \delta_{i j} \mathrm{~d} V+\operatorname{Pr} \int_{V}\left(\phi_{\alpha, i} \phi_{\beta, j}\right) \mathrm{d} V, \\
S_{\alpha i \beta j}^{\prime} & =\frac{\Delta t}{2} U_{i}^{c(n)} U_{j}^{(n)} \int_{V}\left(\phi_{\alpha, k} \phi_{\beta, k}\right) \delta_{i j} \mathrm{~d} V+\frac{\Delta t}{2} U_{i}^{c(n)} U_{j}^{c(n)} \int_{V}\left(\phi_{\alpha, i} \phi_{\beta, j}\right) \mathrm{d} V, \\
A_{\alpha \beta}^{\prime} & =\frac{\Delta t}{2} U_{i}^{c(n+1)} U_{j}^{(n+1)} \int_{V}\left(\phi_{\alpha, i} \phi_{\beta, i}\right) \mathrm{d} V .
\end{aligned}
$$

In eqns (47)-(50), $\bar{M}_{\alpha \beta}$ means the lumped mass matrix obtained simply by summing across each row of the consistent mass matrix $M_{\alpha \beta}$, and placing the results in the diagonal. In eqns (60) and (61), $U_{i}^{c(n)}$ means the value of the velocity which is computed at the centroid of each element using $U_{i}^{n}$.

Though eqns (47)-(50) was derived by considering only a single element, the equations for the assemblage of all elements possesses the same form. Thus eqns (47)-(50) constitutes the basic numerical problem for the finite element analysis of this class of problems.

\section{CALCULATION OF STREAM FUNCTION}

A particularly useful variable for a normal representation of 2-D flow is the stream function. This can be evaluated, knowing the velocity distribution from the equation

$$
\psi_{, i i}=-\omega
$$

where $\psi$ is the stream function and $\omega$ is the vorticity. The relationship between velocity and these variables is given by:

$$
\begin{aligned}
& U_{1}=\psi_{, 2} \\
& U_{2}=\psi_{, 1}
\end{aligned}
$$

and

$$
\omega=-\left[U_{1,2}-U_{2,1}\right]
$$

The appropriate boundary condition is $\psi=0$ on all boundaries.

Again the finite element technique and Galerkin's principle can be used with effect. For the same idealization of the domain the following matrix equation can be obtained by adopting the same approach as outlined previously.

$$
A_{\alpha \beta} \psi_{\beta}^{n+1}=H_{\alpha 1 \beta} U_{\beta 1}^{n+1}+H_{\alpha 2 \beta} U_{\beta 2}^{n+1}+\hat{\Sigma}_{2 \alpha}
$$

where, typically,

$$
\begin{aligned}
A_{\alpha \beta} & =\int_{V}\left(\phi_{\alpha, i} \phi_{\beta, j}\right) \mathrm{d} V, \\
H_{\alpha 1 \beta} & =\int_{V}\left(\phi_{\alpha} \phi_{\beta, 2}\right) \mathrm{d} V,
\end{aligned}
$$




$$
\begin{aligned}
H_{\alpha 2 \beta} & =\int_{V}\left(\phi_{x} \phi_{\beta, 1}\right) \mathrm{d} V, \\
\hat{\Sigma}_{2 \alpha} & =\int_{S}\left(\phi_{\alpha} \psi_{, i}^{n+1} \cdot n_{i}\right) \mathrm{d} S .
\end{aligned}
$$

Therefore, once the nodal velocities are known, the stream function and the corresponding "visual" contour plot representation can be obtained.

\section{DIMENSIONLESS HEAT TRANSFER COEFFICIENT}

An average Nusselt number for the cavity can be calculated from:

$$
\bar{N} u=\frac{h_{X_{2}} H}{K}=\int_{0}^{H / H^{\prime}}\left(\frac{\partial \Theta}{\partial X_{1}}\right)_{X_{1}=0} \mathrm{~d} X_{2}
$$

and

$$
\bar{N} u_{w}=\frac{\bar{h}_{x_{2}} W}{K}=\frac{1}{H / w} \bar{N} u
$$

where $\bar{h}_{X_{2}}$ is the average heat transfer coefficient, $\vec{N} u_{w}$ is the average Nusselt number and $w$ is the significant length.

\section{NUMERICAL EXAMPLES}

\subsection{Flow in a driven cavity}

The driven cavity flow is taken as the first application of the proposed scheme. The problem definition is given in Fig. 1 . The spatial discretization consists of nonuniform meshes of 1800 (for $\mathrm{Re}=0$ and 1000) and 3200 (for $\mathrm{Re}=5000$ ) three-noded triangular elements. A pressure datum $P=0$ is specified at the middle of the bottom wall. Iso-streamlines and pressure contours at steady state are plotted in Figs 2-4 for $R e=0,1000,5000$, compares fairly well with the results obtained by Yang and Atluri $[4,5]$. The plot of the streamlines for $R e=1000$ and 5000 clearly indicates the presence of two secondary vortices at the lower corners of the cavity and three secondary vortices at the lower corners and upper left corner of the cavity as reported by these and other authors. The computation was performed in single precision ( 32 bits per word) and $0.4 \mathrm{MB}$ were used for all cases at the Tokyo University computer center ( $\mathrm{M} 680 \mathrm{H}$ computer). The CPU time was $50 \mathrm{~s}$ for $\mathrm{Re}=0 ; 4 \mathrm{~min}$ for $\mathrm{Re}=1000$; and $22 \mathrm{~min} 10 \mathrm{~s}$ for $\mathrm{Re}=5000$.

The pressure fields are found to be completely free from chequerboard oscillations. No pressure splitting is encountered with the present formulation, even when using purely Dirchlet-type boundary conditions.

The profiles of the horizontal velocity along the vertical center line of the driven cavity $\left(X_{1}=0.5\right)$ and the vertical velocity along the horizontal center line of the driven cavity $\left(X_{2}=0.5\right)$ is illustrated for $\mathrm{Re}=1000$ in Fig. 5. They show almost exact agreement with those of Ghia et al. [6]. Finally, a comparison of the properties of the primary and secondary vortices in the driven cavity flow is provided in Table 1 . In general the agreement between the present solutions and other numerical solutions are excellent.

\subsection{Sudden expansion problem}

The second example is a rearward facing step. This problem has been treated in numerous previous studies including both experimental and computational work. In addition, this problem is cited in Ref. [7] as one that is susceptible to producing spurious pressure modes. The problem definition is given in Fig. 6, which shows an upward facing step of expansion ratio (1:2). At the top as well as bottom walls, the boundary conditions are $U_{1}=U_{2}=0$. At the inlet the boundary conditions are $U_{1}=240 X_{2}\left(1-X_{2}\right)$ and $U_{2}=0$ with the origin of $X_{2}$ as shown in Fig. 6 . The channel inlet and outlet is one and two units high, respectively. A parabolic velocity profile is described at the inlet resulting in a Reynolds number of 60 , while low is large enough to cause the 


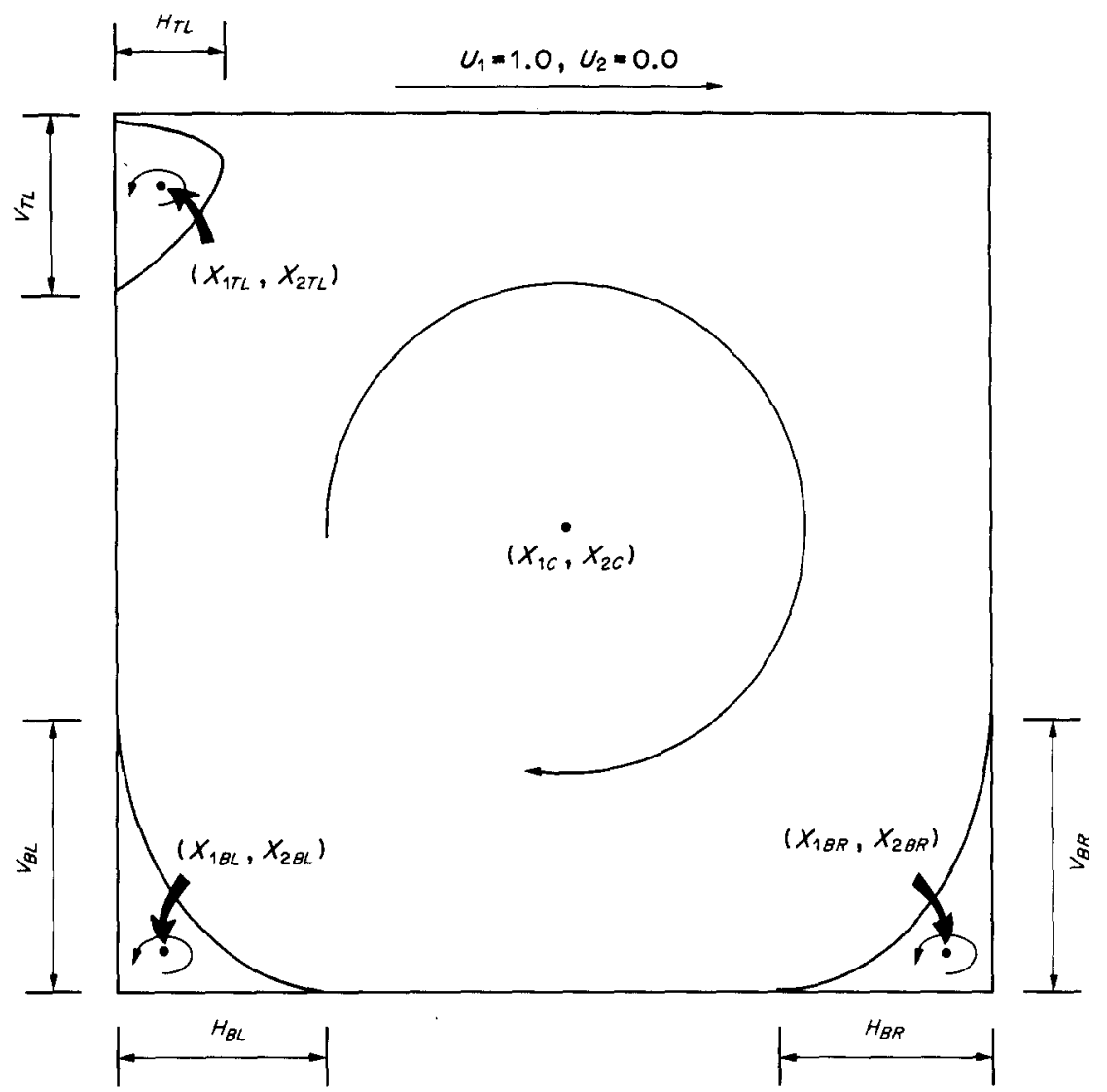

Fig. 1. Cavity flow configuration, coordinates, nomenclature and boundary conditions.

development of a significant recirculation region in the expansion corner. The computing conditions are as follows: $\Delta t=0.005, v=1.0$. Computed steady state velocity vectors, streamlines and pressure contours are plotted in Fig. 7. The computation was performed in single precision on the Tokyo University computer center $\mathrm{M} 680 \mathrm{H}$ computer. The used memory size was $0.2 \mathrm{MB}$ and CPU time was $54 \mathrm{~s}$ for 1000 time steps.

Qualitatively, one would expect the pressure to be nearly uniform across the inlet section, being consistent with the imposed fully developed inlet conditions. Further downstream, steep negative pressure gradients are developed in the narrow portion of the channel followed by adverse pressure gradients in the wider section. This general pattern is depicted in the pressure contours as shown in Fig. 7(c). A plot of the pressure at the bottom wall is shown in Fig. 8. The results have been compared with those obtained by Lee et al. [8]. Both results are extremely well in agreement.

\subsection{Flow over a square step}

The third example is more complex in terms of both the geometry and the predicted flow field. The problem definition is given in Fig. 9. It is to be remarked that this problem has been analysed well by several investigators $[5,9,10]$. The flow is considered in a 1 unit high channel consisting of a step located at 1.2 units from the inlet which is 0.4 units high and 0.4 units across. The inlet boundary condition is $U_{1}=1, U_{2}=0$. The length of the channel is assumed to be 4 units from the inlet.

The presently computed results for velocity vectors, pressure contours and streamlines at $\operatorname{Re}=85$, and at times $t=1.0,2.0,3.0$ and 4.0 units, respectively are shown in Figs 10-13. The computing conditions are as follows: $\Delta t=0.0025, v=0.0118$. The CPU time was $1 \mathrm{~min} 30 \mathrm{~s}$ for 2000 time steps using $\mathrm{M} 680 \mathrm{H}$ computer at Tokyo University computer center. The eddy length at $t=4.0$, shown in Fig. 13, is in excellent agreement with the results reported by Yang and Atluri [5]. 


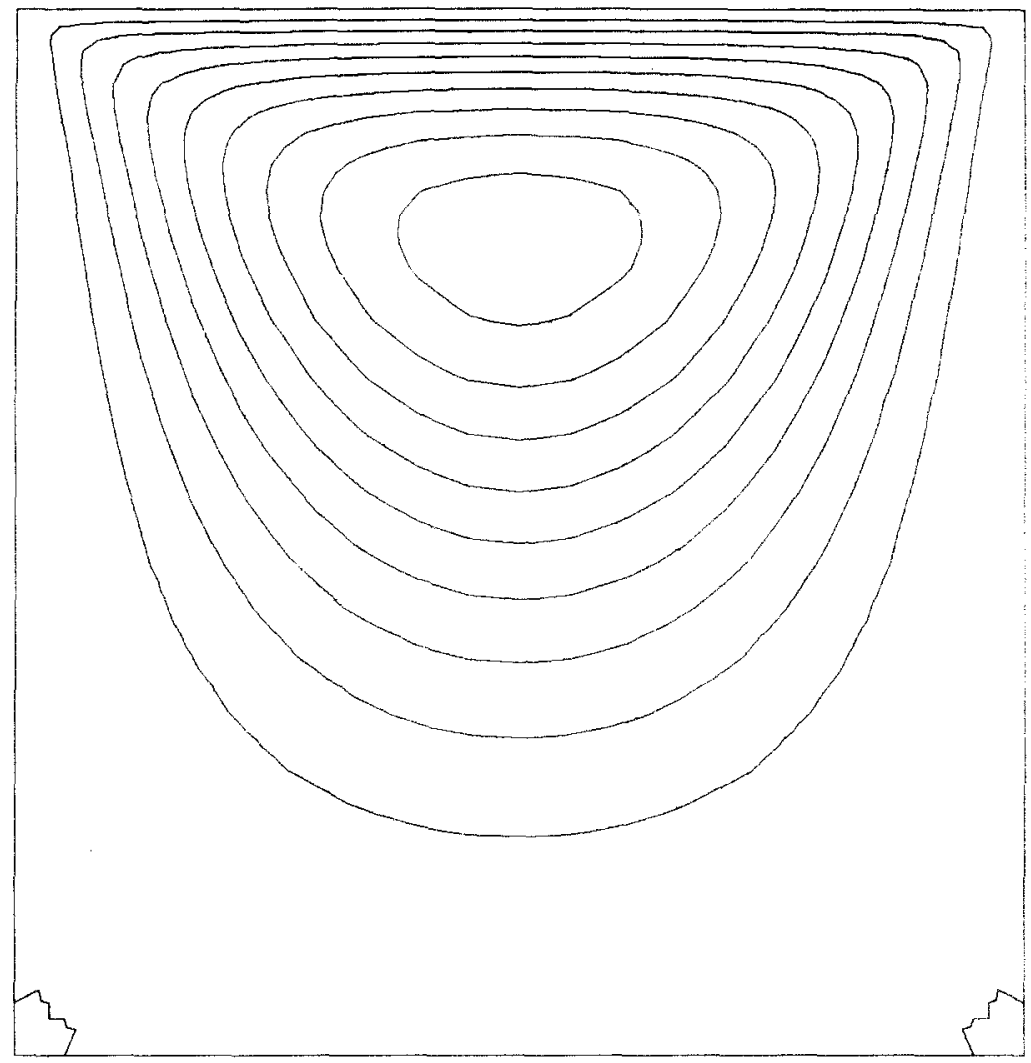

(a)

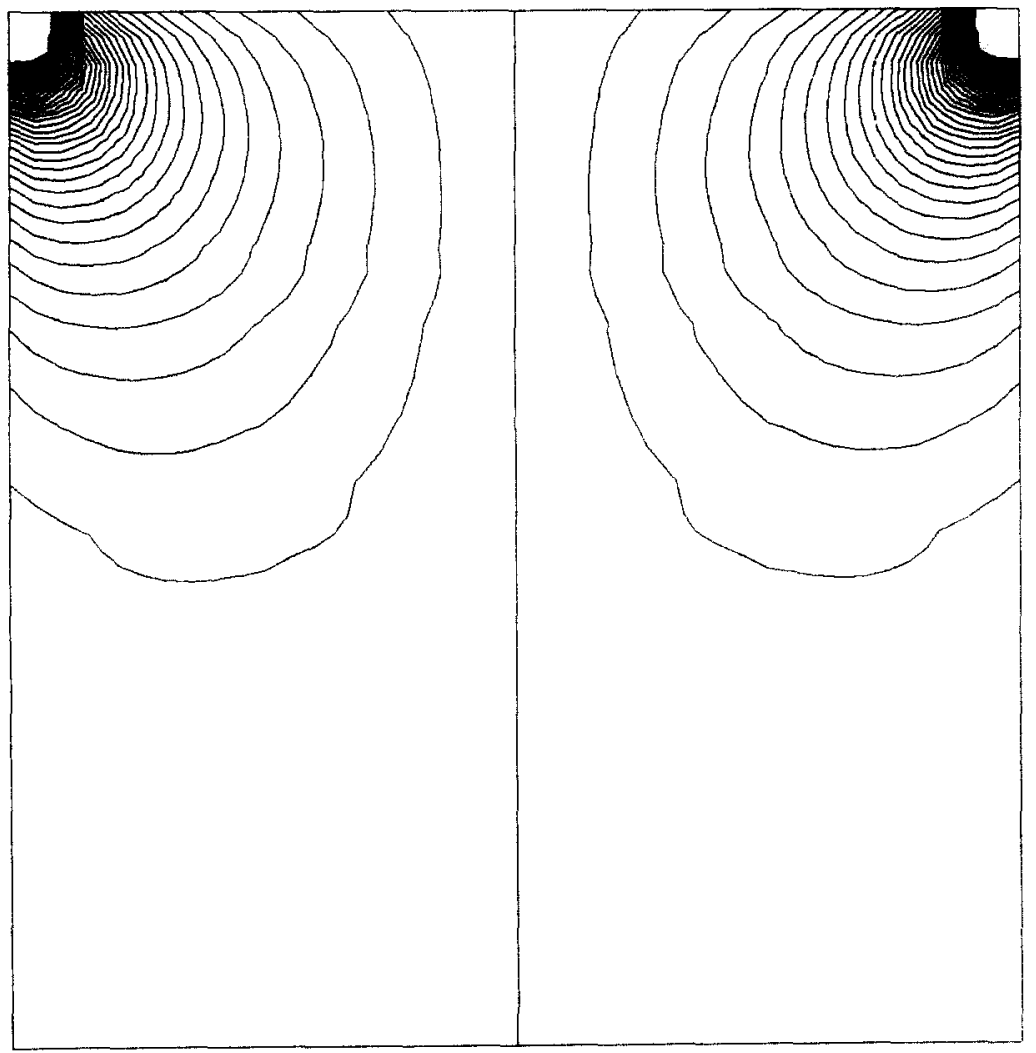

(b)

Fig. 2. Driven cavity flow at $R e=0$. (a) Streamlines; (b) pressure contours. 


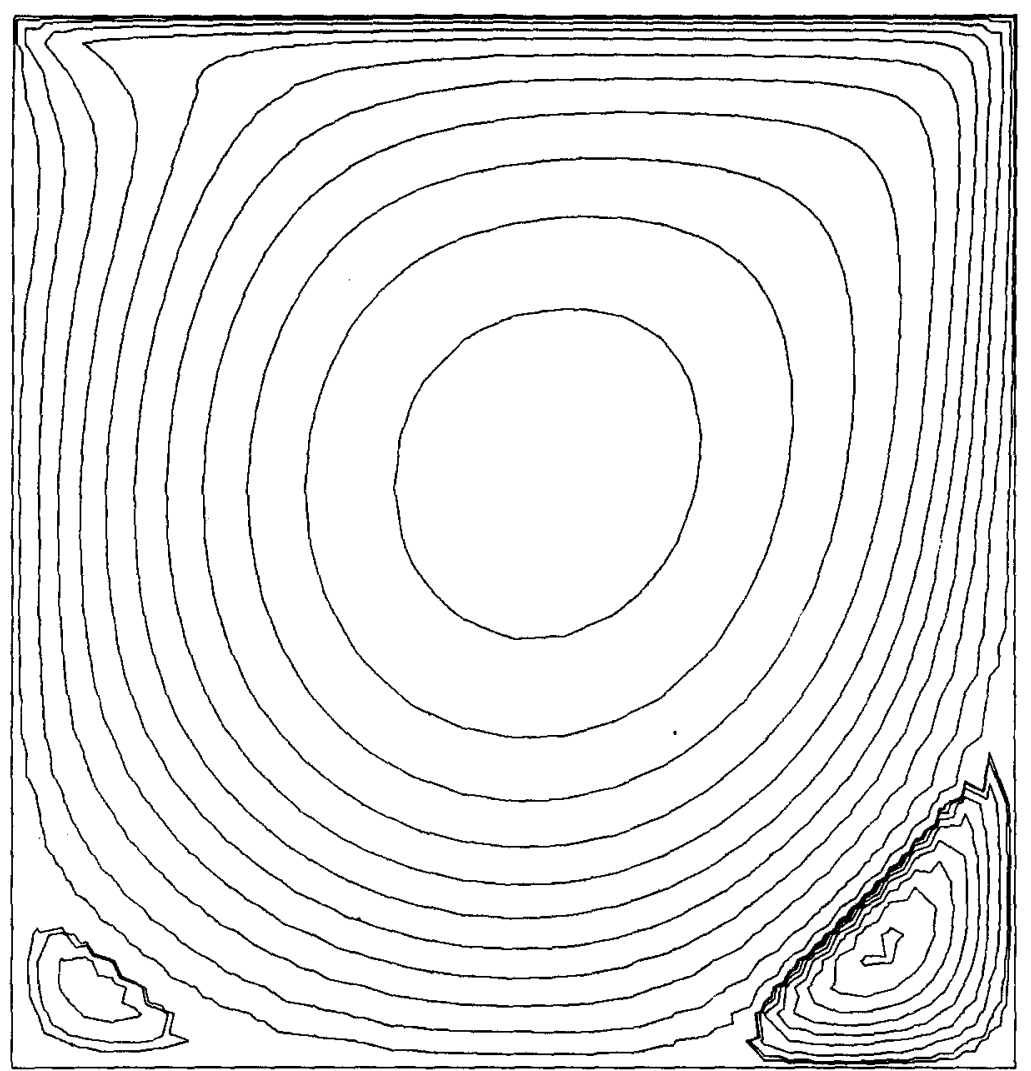

(a)

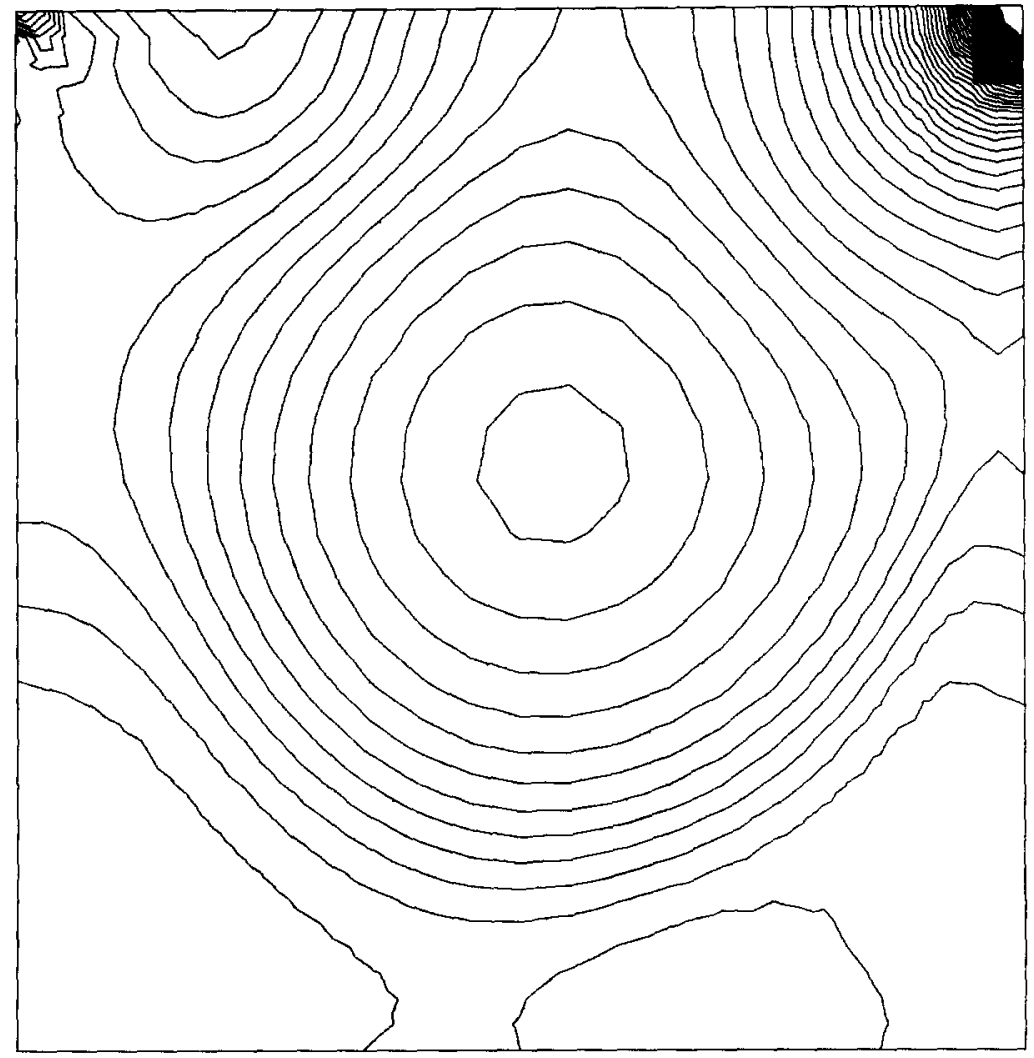

(b)

Fig. 3. Driven cavity flow at $R e=1000$. (a) Streamlines; (b) pressure contours. 


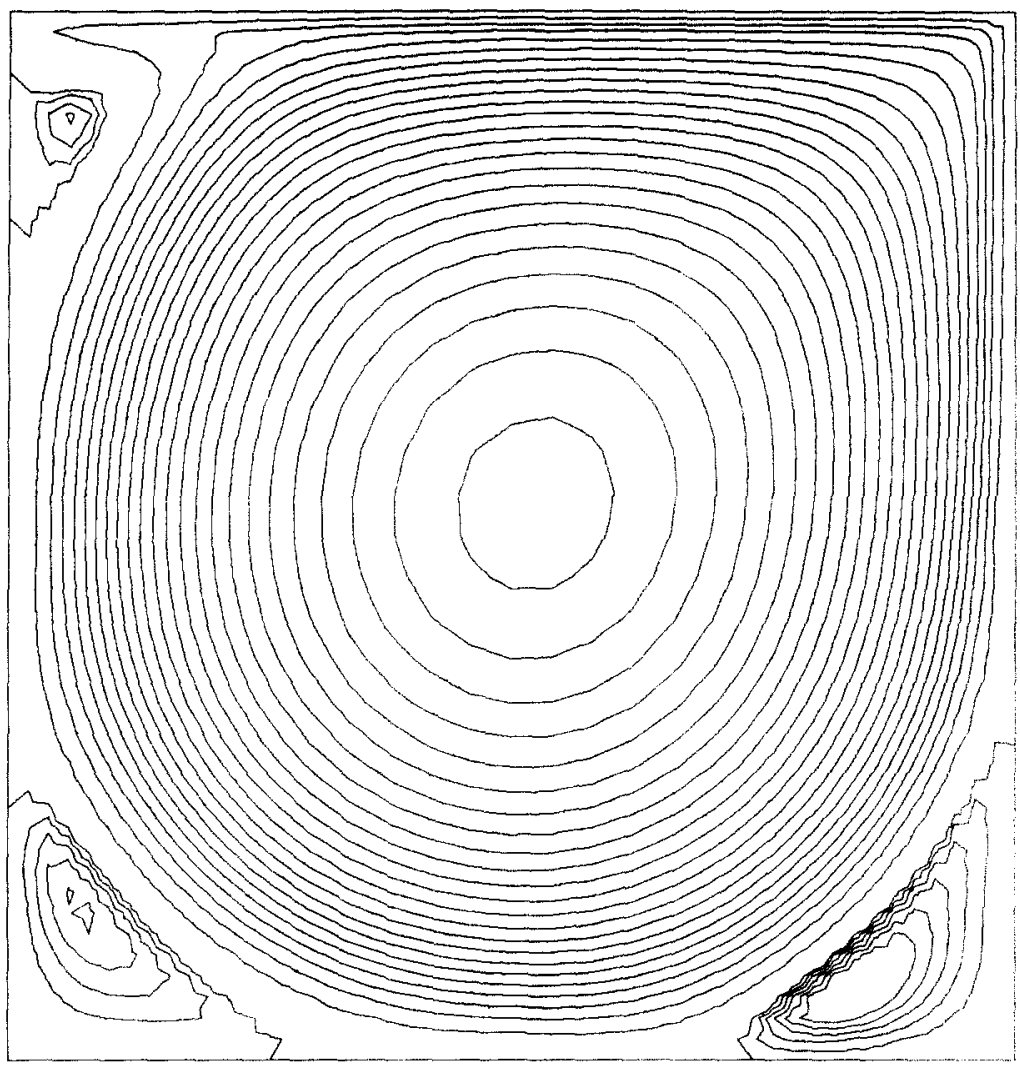

(a)

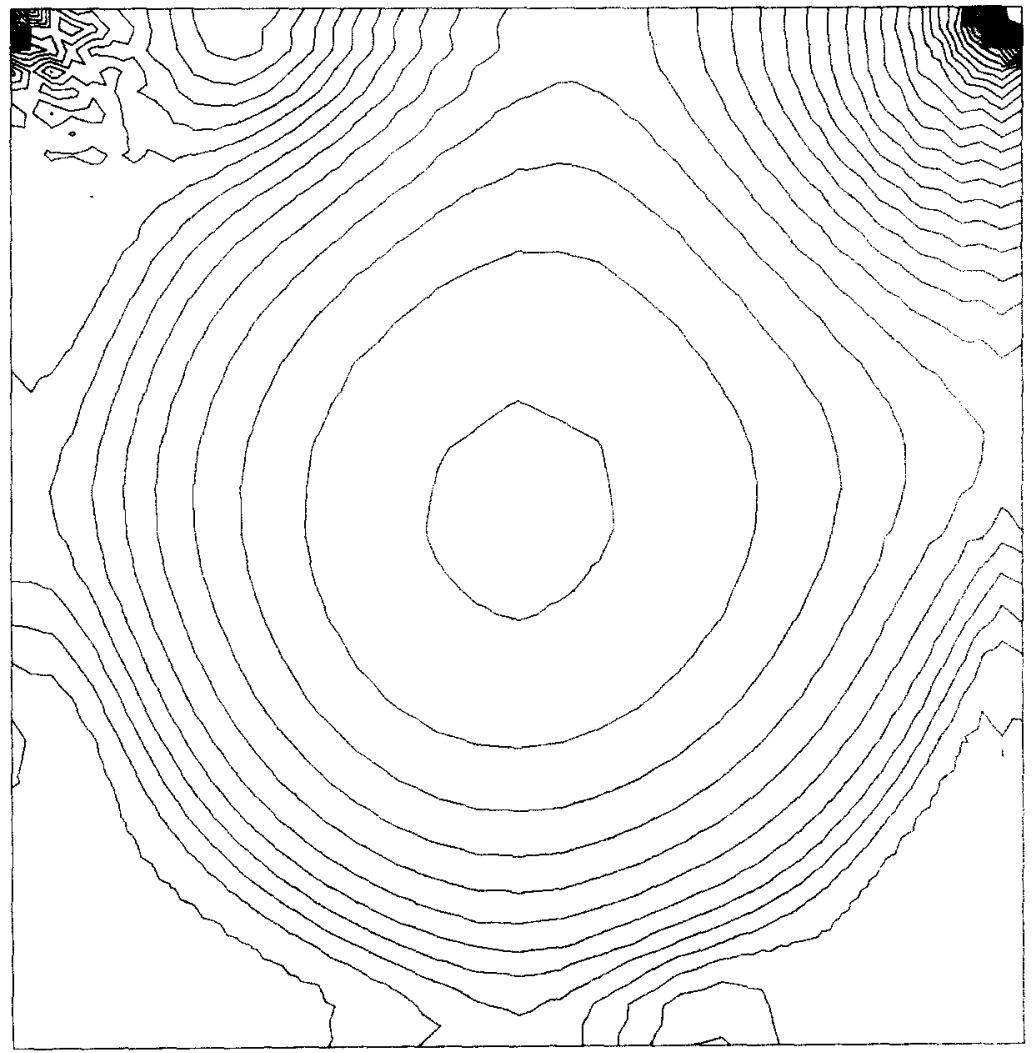

(b)

Fig. 4. Driven cavity flow at $\operatorname{Re}=5000$. (a) Streamlines; (b) pressure contours. 


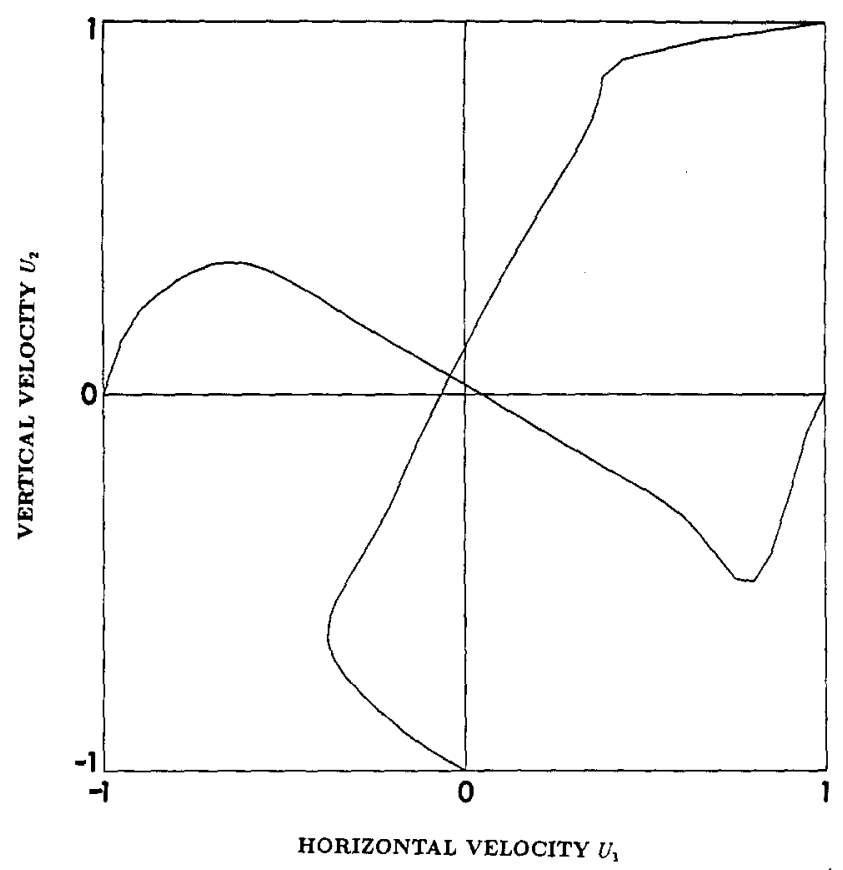

Fig. 5. Velocity profiles along horizontal and vertical center lines at $\operatorname{Re}=1000$.

\subsection{Flow around a flat plate}

The fourth problem is a flow around a flat plate. The problem statement is depicted in Fig. 14. The flow is considered in a 2.5 units high channel consisting of a flat plate located at 0.8 units from the inlet which is 1 unit high. The inlet boundary condition is $U_{1}=1, U_{2}=0$. The length of the channel is assumed to be 2.5 units from the inlet.

The presently used uniform finite element mesh for $\mathrm{Re}=100$ consists of 3160 three-noded triangular elements and 1600 nodal points. The computing conditions are as follows: $\Delta t=0.01$, $v=0.01$. The used memory size was $0.4 \mathrm{MB}$ and CPU time was $32 \mathrm{~s}$ for 100 time steps using $\mathrm{M} 680 \mathrm{H}$ computer at Tokyo University computer center. Presently computed results for velocity vectors, pressure and streamlines at $\operatorname{Re}=100$, and at times $t=0.25,0.5$ and 1.0 , respectively are shown in Figs 15-17. The computed results are good in agreement with the appearance observed in the experiment and other numerical simulations.

Table 1. Properties of primary and secondary vortices

\begin{tabular}{cccc}
\hline Re & & Present method & Ghia et al. $[6]$ \\
\hline 1000 & $\left(X_{1 C}, X_{2 C}\right)$ & $(0.5326,0.5631)$ & $(0.5313,0.5625)$ \\
& $\left(X_{1 B R}, X_{2 B R}\right)$ & $(0.8612,0.1108)$ & $(0.8594,0.1094)$ \\
& $\left(X_{1 B L}, X_{2 B L}\right)$ & $(0.0845,0.0769)$ & $(0.0859,0.0781)$ \\
& $H_{B R}$ & 0.3072 & 0.3034 \\
& $V_{B R}$ & 0.3483 & 0.3536 \\
& $H_{B L}$ & 0.2108 & 0.2188 \\
& $V_{B L}$ & 0.1623 & 0.1680 \\
& $\left(X_{1 C}, X_{2 C}\right)$ & $(0.5120,0.5347)$ & $(0.5117,0.5352)$ \\
& $\left(X_{1 B R}, X_{2 B R}\right)$ & $(0.8104,0.0751)$ & $(0.8086,0.0742)$ \\
& $\left(X_{1 B L}, X_{2 B L}\right)$ & $(0.0738,0.1371)$ & $(0.0703,0.1367)$ \\
& $\left(X_{1 T L}, X_{2 T L}\right)$ & $(0.0638,0.9091)$ & $(0.0625,0.9102)$ \\
& $H_{B R}$ & 0.3527 & 0.3565 \\
& $V_{B R}$ & 0.4203 & 0.4180 \\
& $H_{B L}$ & 0.3162 & 0.3184 \\
& $V_{B L}$ & 0.2668 & 0.2643 \\
& $H_{T L}$ & 0.1208 & 0.1211 \\
& $V_{T L}$ & 0.2665 & 0.2693 \\
\hline
\end{tabular}




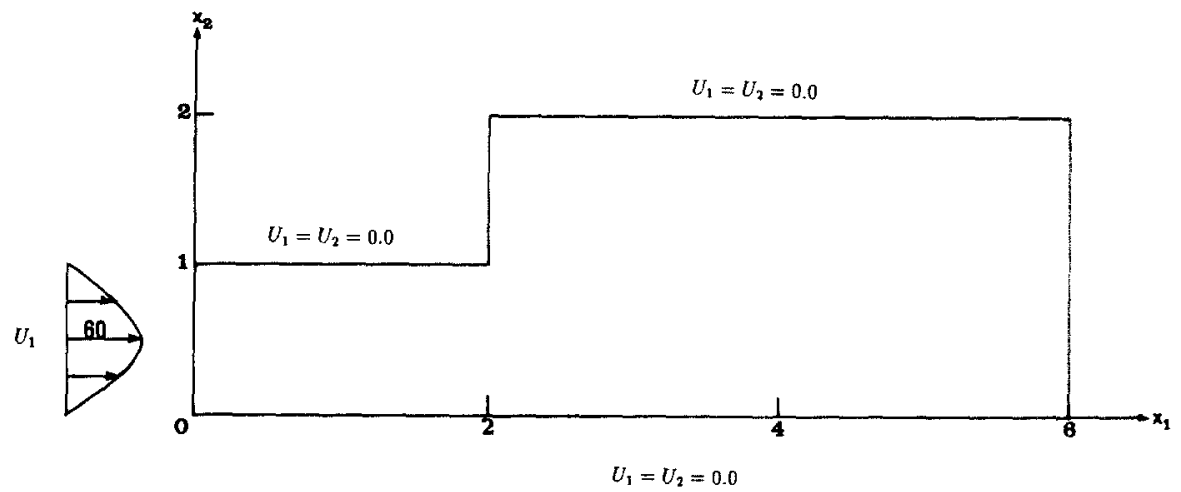

(a)

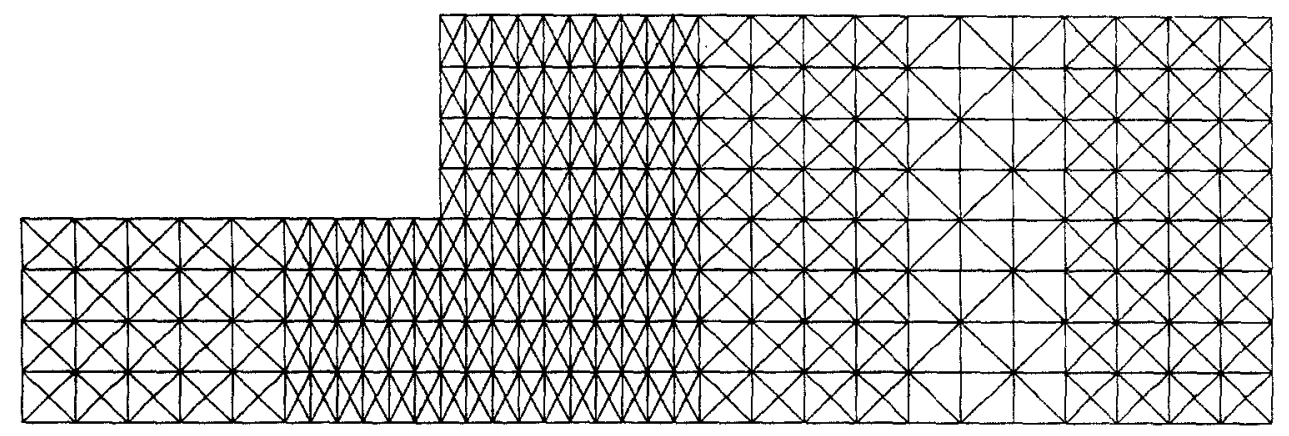

(b)

Fig. 6. Flow through sudden enlargement. (a) Problem description; (b) finite element mesh.

\subsection{Flow around a stationary sphere}

The fifth problem is the axisymmetric flow past a rigid stationary sphere. The problem definition is given in Fig. 18. Computed steady state velocity vectors and pressure contours for $\operatorname{Re}=10$ are reported in Fig. 19. The pressure contours for $\mathrm{Re}=10$ are almost indistinguishable from those reported by other researchers. In Fig. 20 the distribution of pressure and vorticity around the sphere is depicted and compared with the infinite-domain semi analytical results [11]. Ahead of the sphere, the solution for both Reynolds number are equally accurate, whereas in the recirculating region behind the sphere, the agreement is better for the higher values of the Reynolds number. This is presumably a consequence of the finite extent of the computational domain which is more relevant when the region of dominant viscous effects is larger. The CPU time for this problem was $8 \mathrm{~s}$ to get a steady state solution using $\mathrm{M} 680 \mathrm{H}$ computers at Tokyo University computer center.

\subsection{Natural convection in rectangular enclosures}

The final example is concerned with the laminar, 2-D motion (generated by a temperature gradient) of an incompressible fluid in a rectangular enclosure. The left and right vertical walls have a specified temperature of -0.5 and 0.5 , respectively, and top and bottom walls are either insulated or subjected to a linear variation of temperature. All of the boundary conditions on the velocity field are of essential type. The problem description is shown in Fig. 21.

7.6.1. Isothermal plots. To illustrate the effect of the Rayleigh number and Prandtl number $\operatorname{Pr}=0.71$ and aspect ratio $H / W=1.0$, the isotherm lines for insulated temperature boundary conditions for increasing values of $\mathrm{Ra}$, upto $10^{7}$, are shown in Fig. 22. For low $\mathrm{Ra} \leqslant 10^{3}$ the isotherm lines are almost linear everywhere inside such that $\Theta=X_{1}$, i.e. heat transfer is by conduction. As the Rayleigh number increases, the convective effects become more apparent and the isotherm lines progressively distort. These distortions result in high temperature gradient near the wall. Near the extremities the patterns are strongly influenced by the temperature condition 


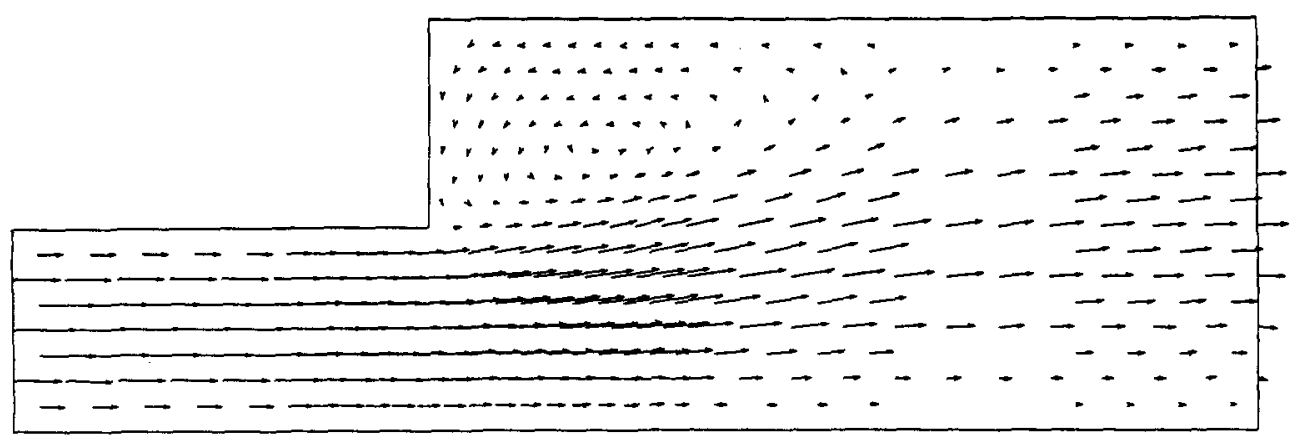

(a)

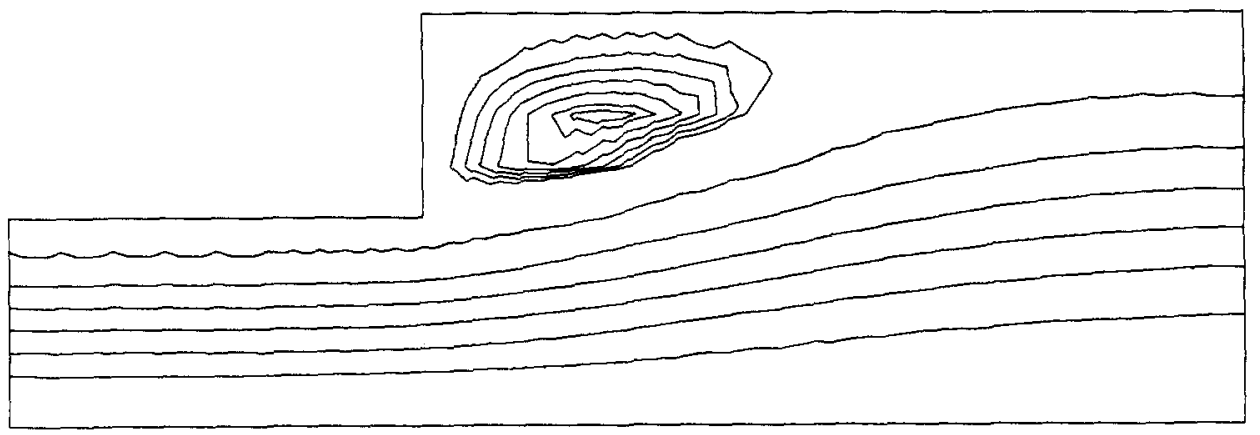

(b)

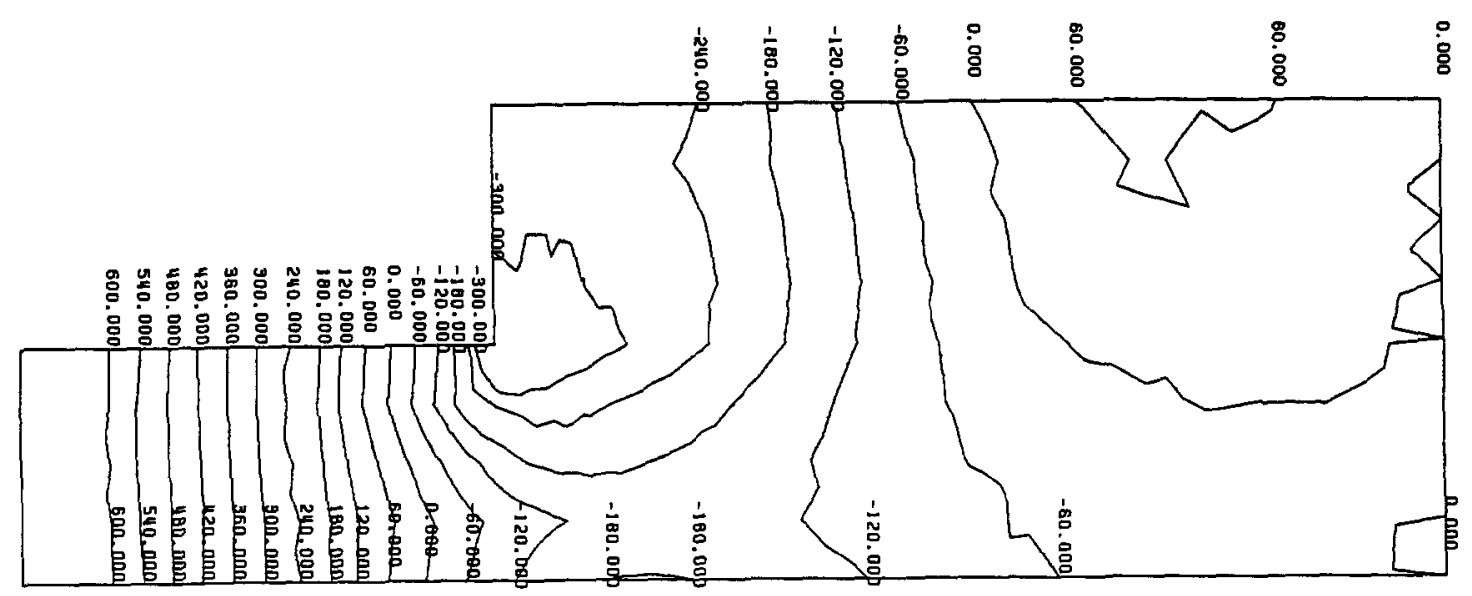

(c)

Fig. 7. Computed results for flow through sudden enlargement at $\operatorname{Re}=60$. (a) Velocity vectors; (b) streamlines; (c) pressure contours.

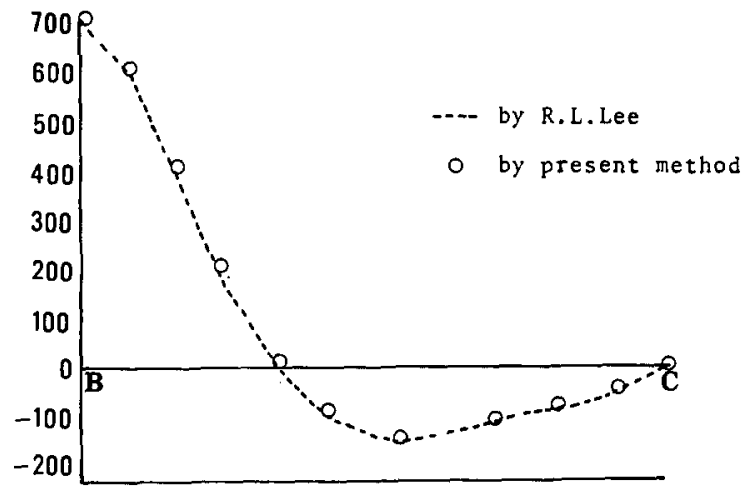

Fig. 8. Pressure profiles along the bottom wall $\left(X_{2}=0\right)$ of the flow through sudden enlargement. 


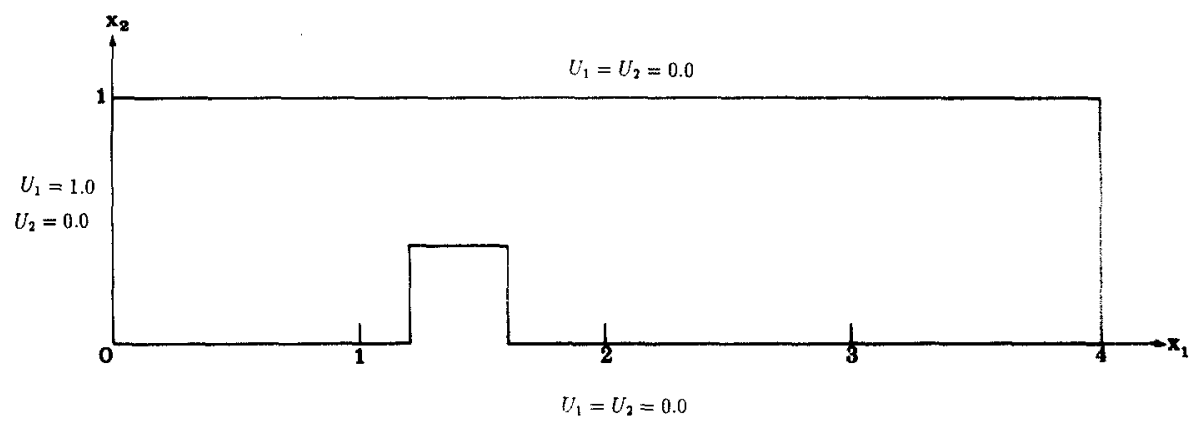

(a)

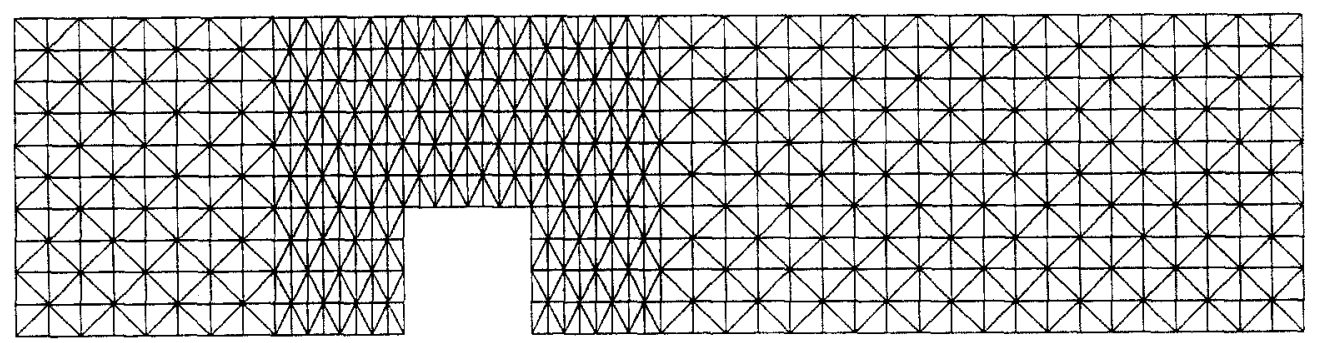

(b)

Fig. 9. Flow over a square step. (a) Probjem description; (b) finite element mesh.

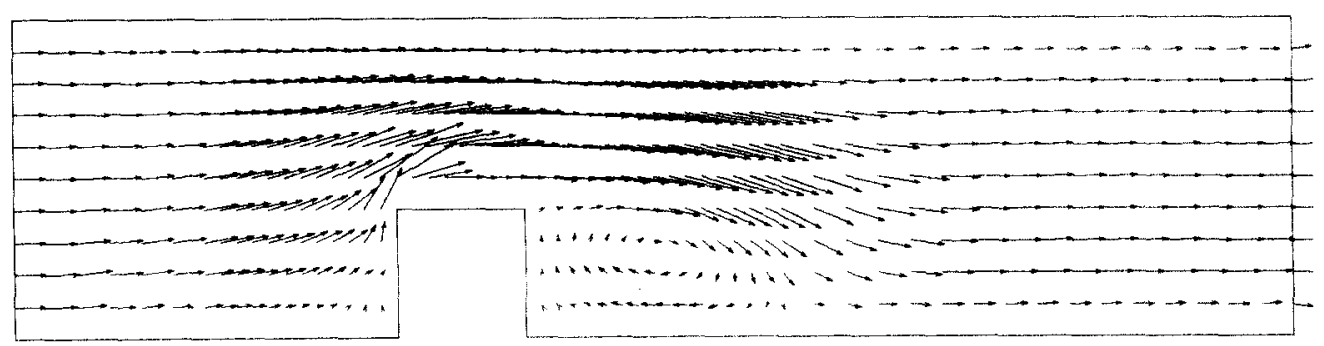

(a)

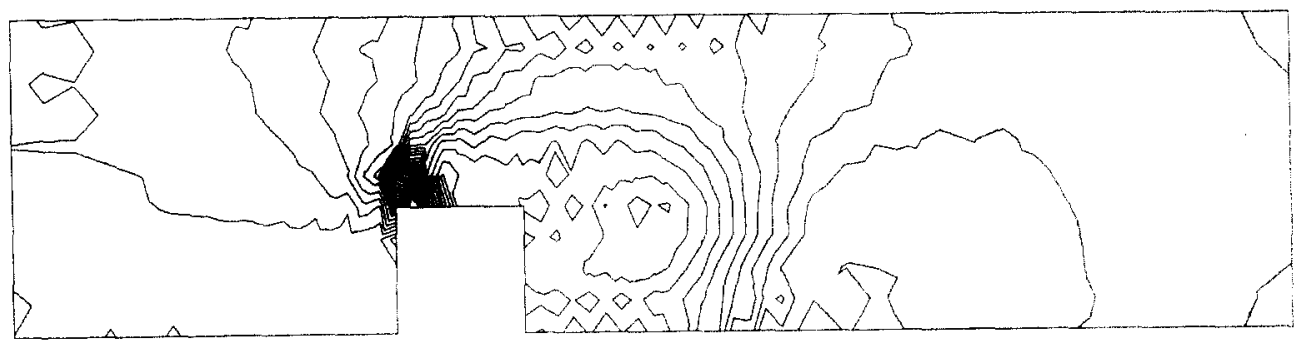

(b)

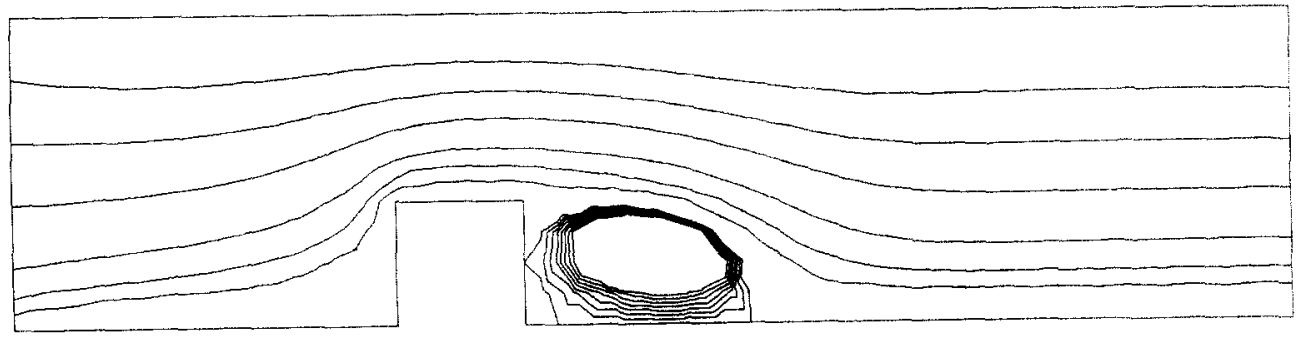

(c)

Fig. 10. Unsteady flow at $\operatorname{Re}=85, t=1,0$. (a) Velocity vectors; (b) pressure contours; (c) streamlines 


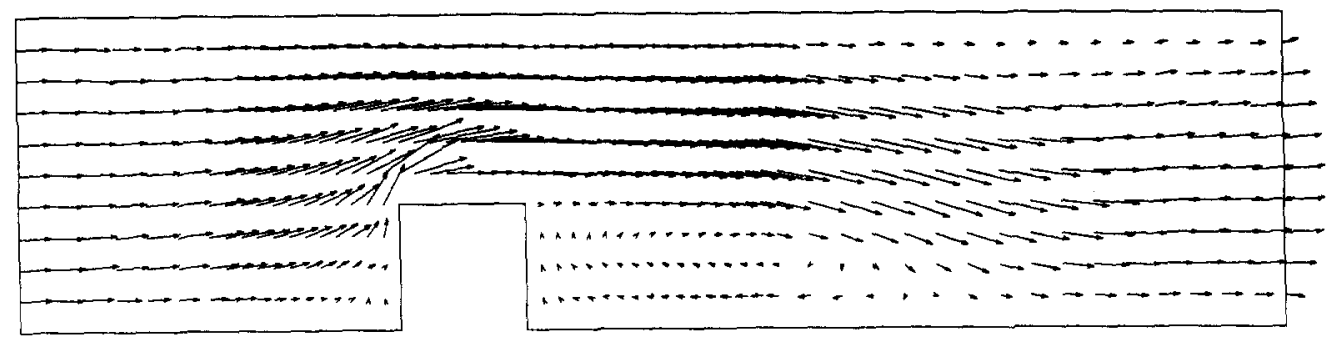

(a)

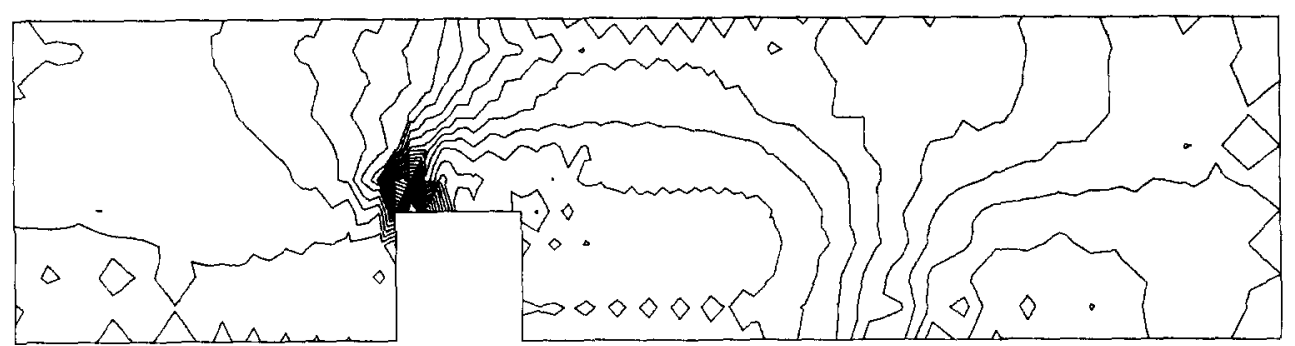

(b)

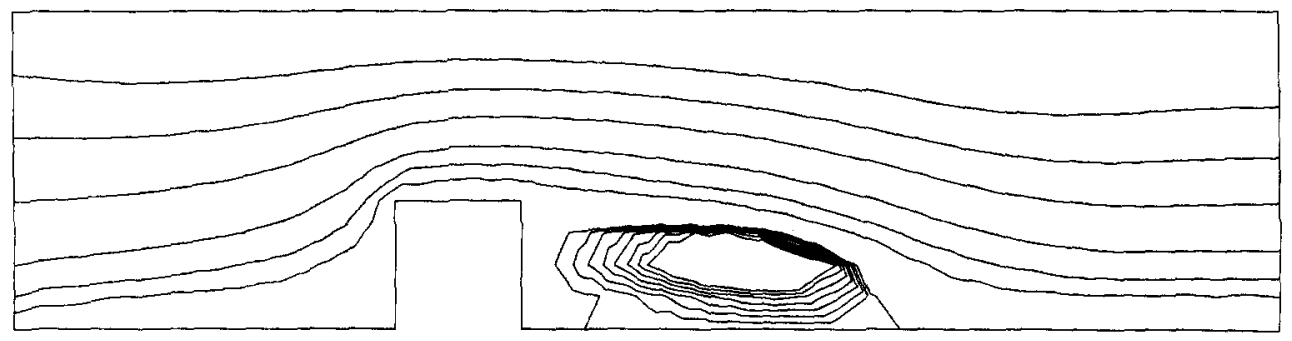

(c)

Fig. 11. Unsteady flow at $\operatorname{Re}=85, t=2.0$. (a) Velocity vectors; (b) pressure contours; (c) streamlines. 


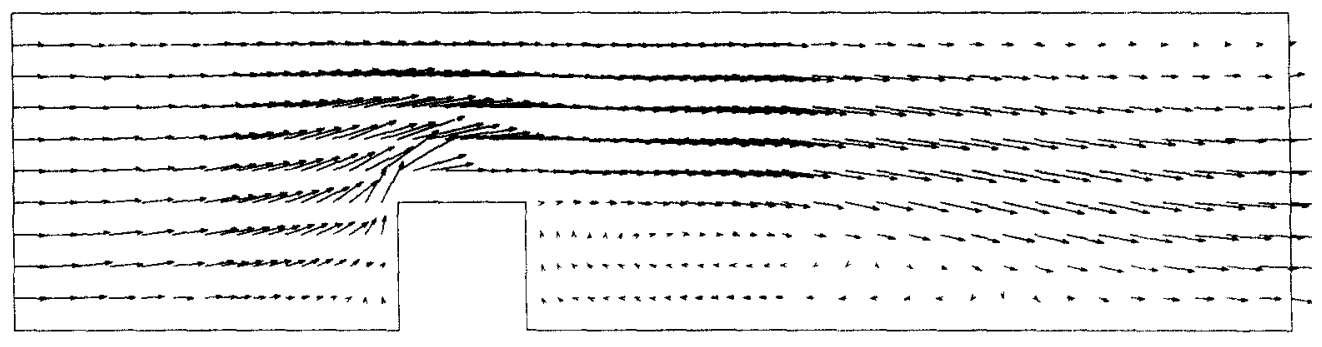

(a)

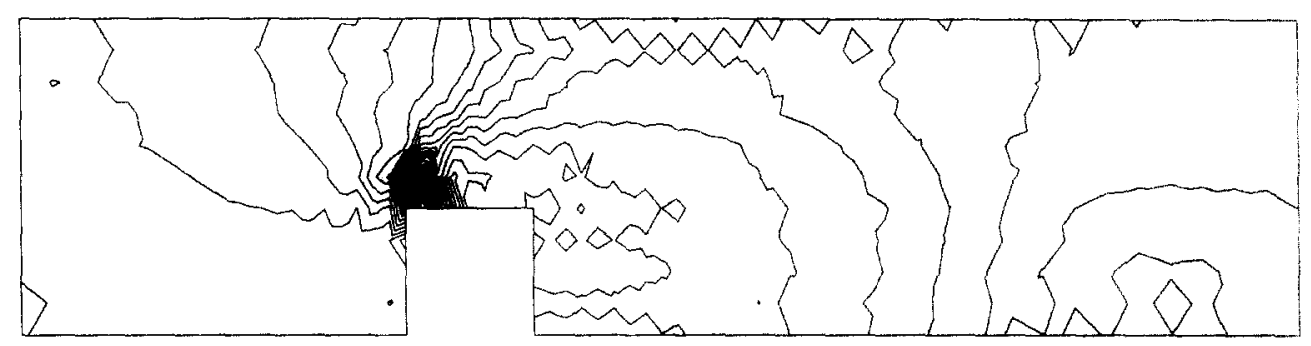

(b)

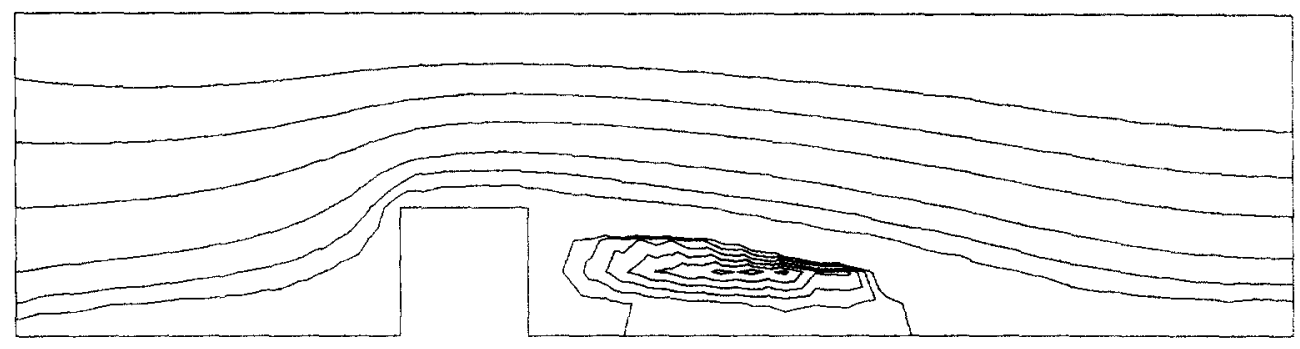

(c)

Fig. 12. Unsteady flow at $\operatorname{Re}=85, t=3.0$. (a) Velocity vectors; (b) pressure contours; (c) streamlines. 


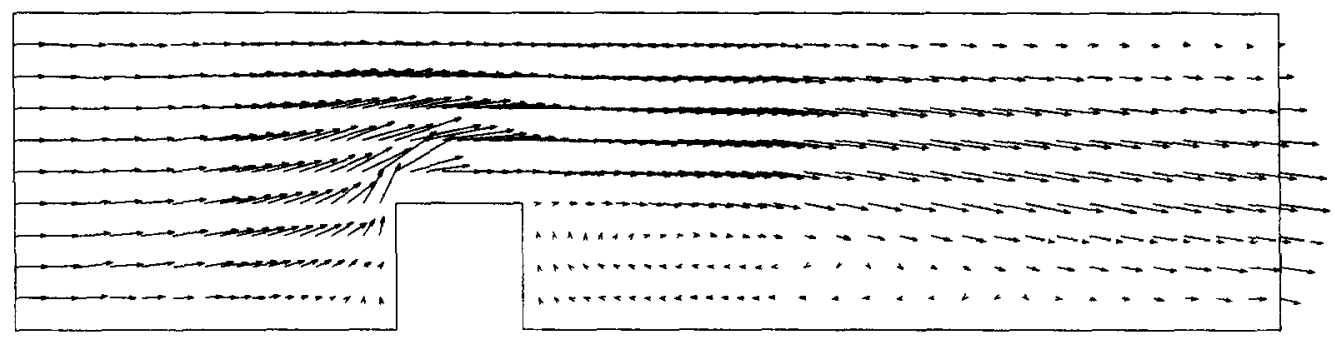

(a)

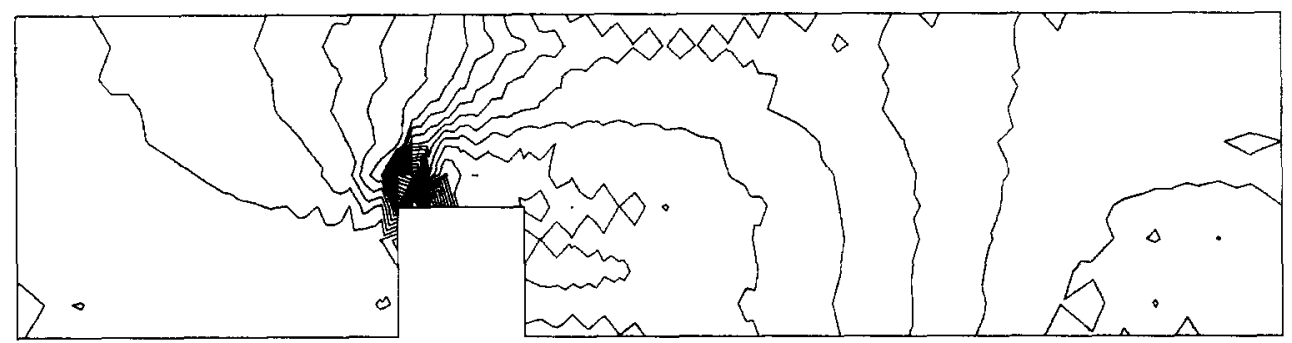

(b)

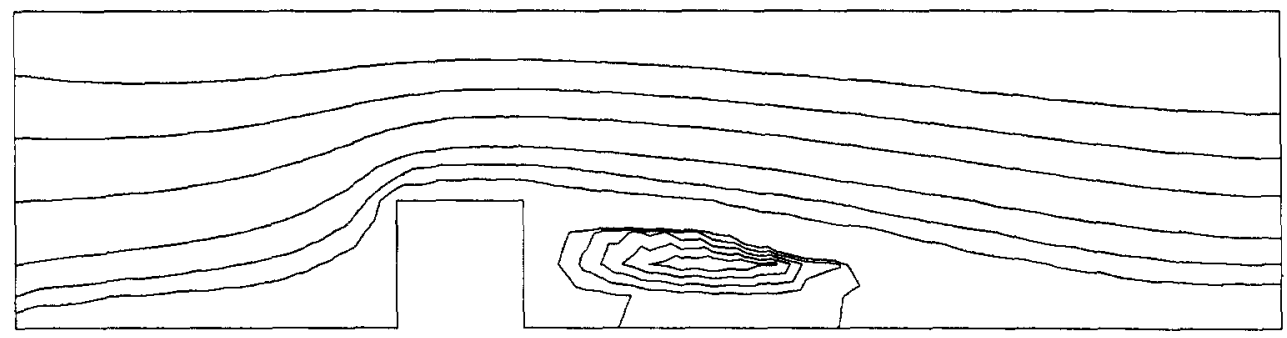

(c)

Fig. 13. Unsteady flow at $\operatorname{Re}=85, t=4.0$. (a) Velocity vectors; (b) pressure contours; (c) streamlines. 


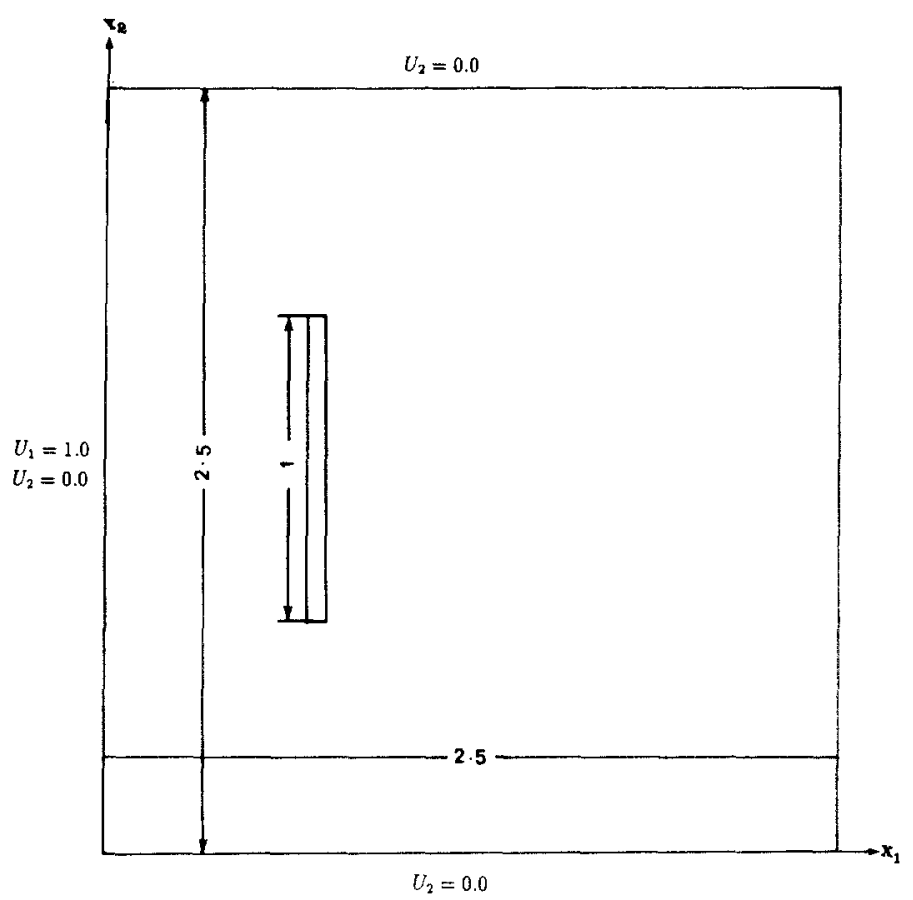

(a)

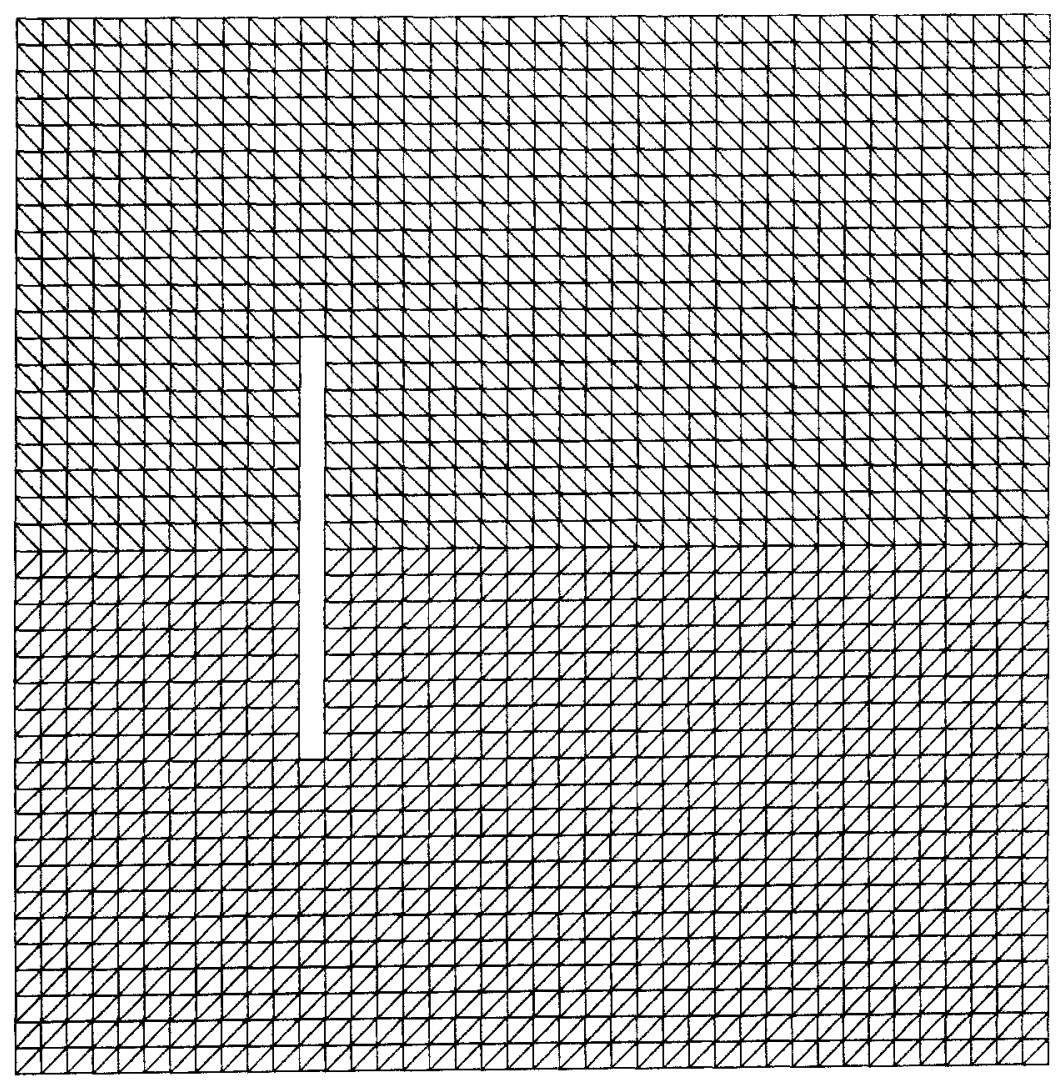

(b)

Fig. 14. Flow around a flat plate. (a) Problem description; (b) finite element mesh. 


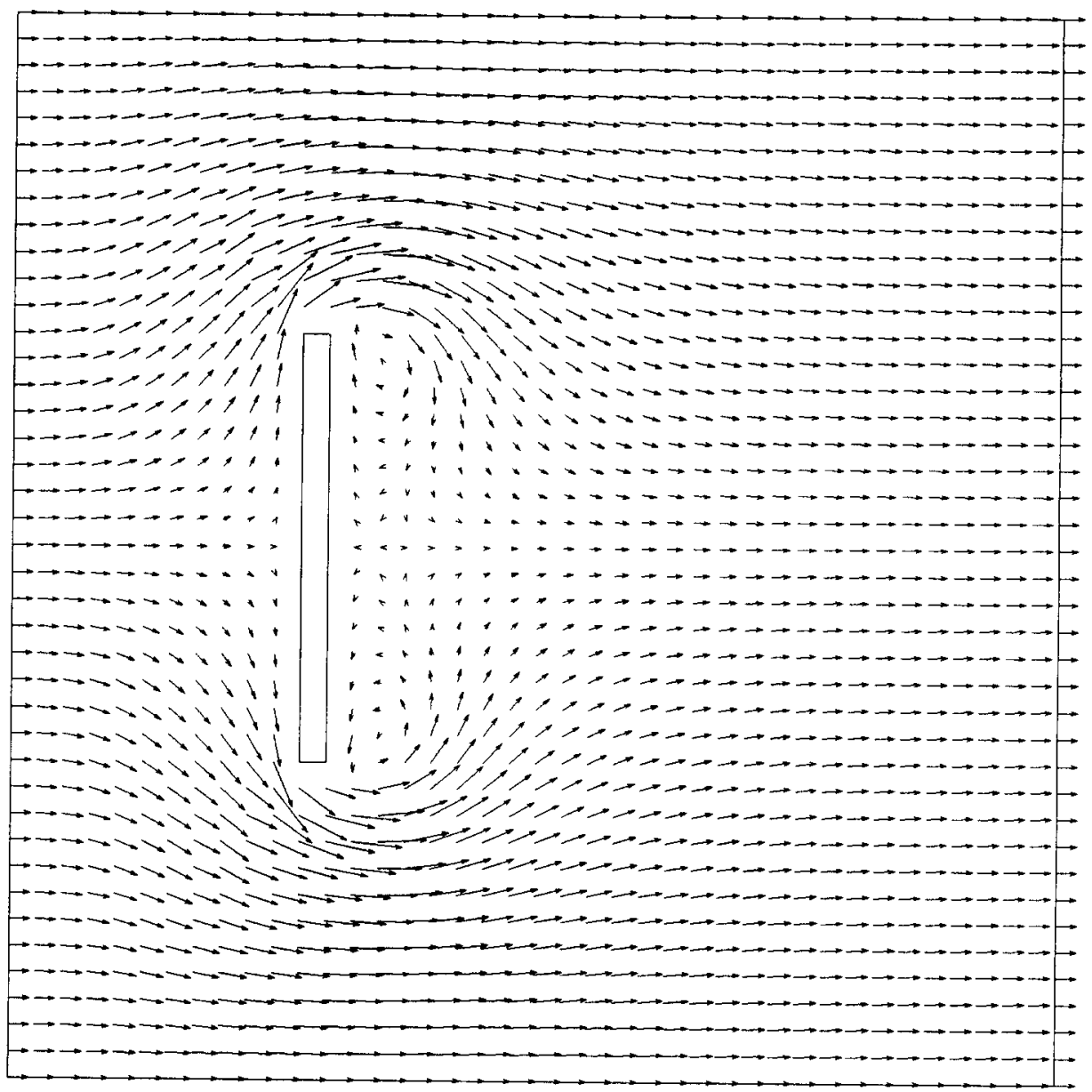

Fig. 15(a) 


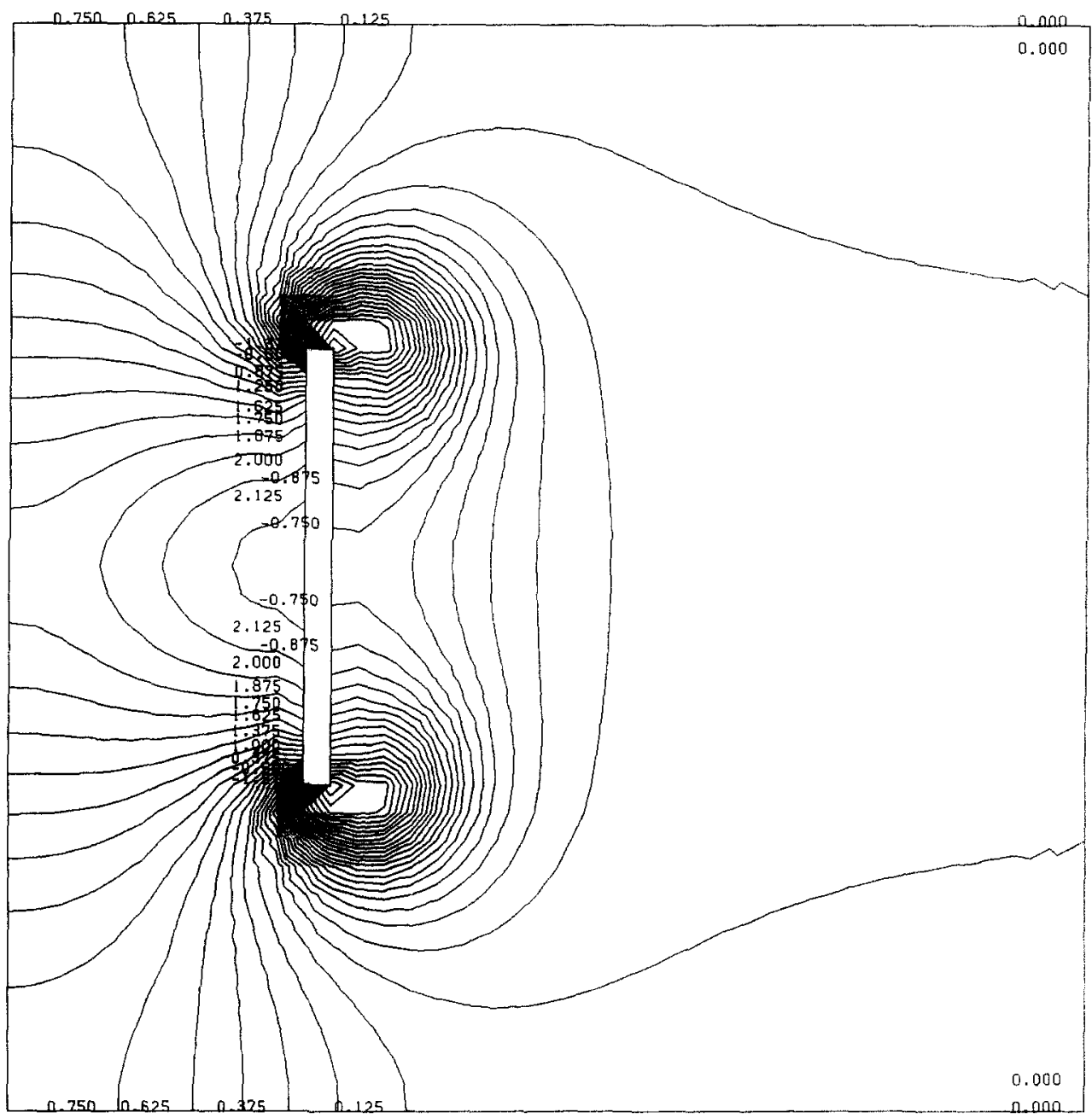

Fig. 15(b) 


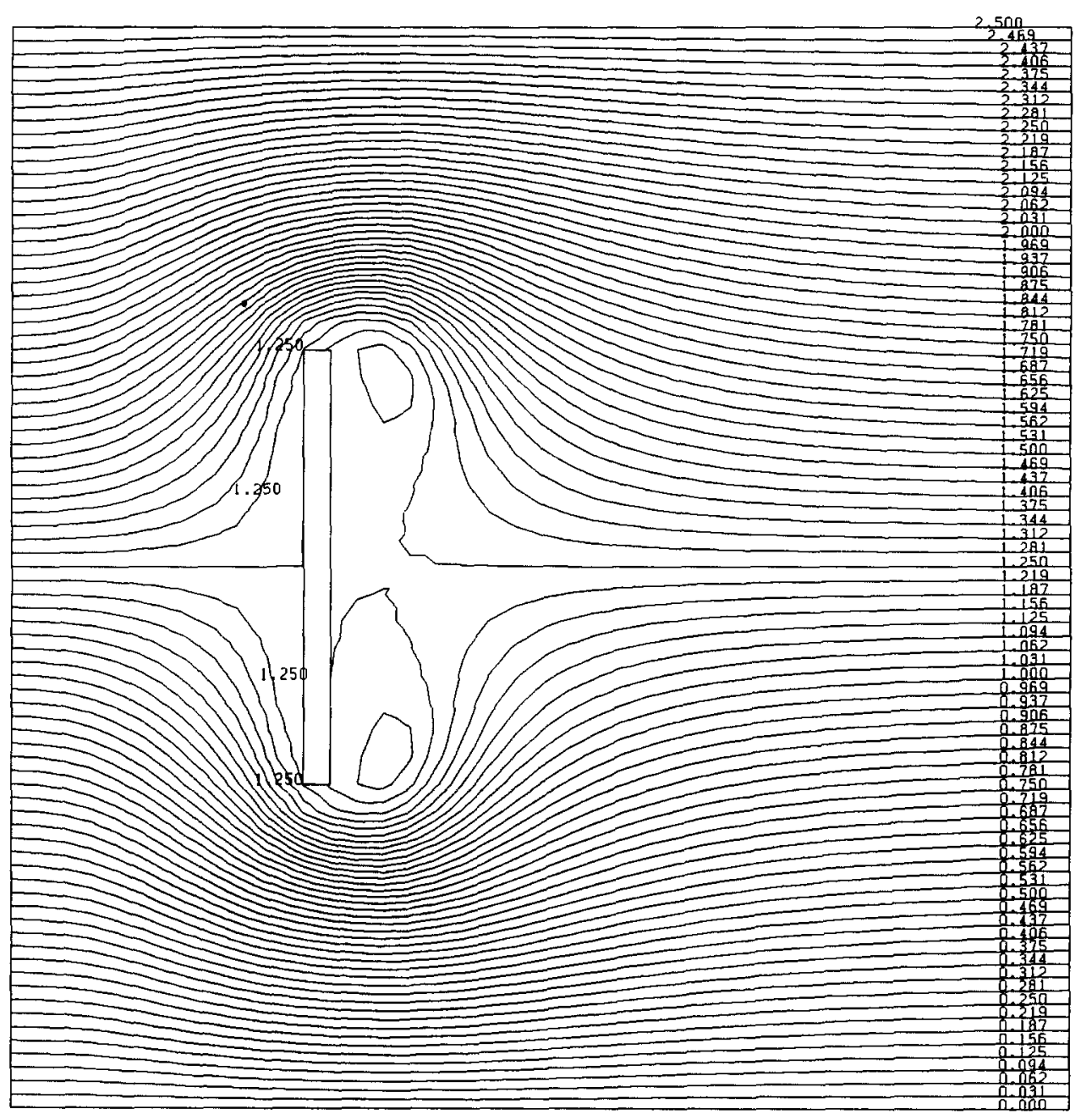

Fig. 15(c)

Fig. I5. Unsteady flow at $\operatorname{Re}=100, t=0.25$. (a) Velocity vectors; (b) pressure contours; (c) streamlines. 


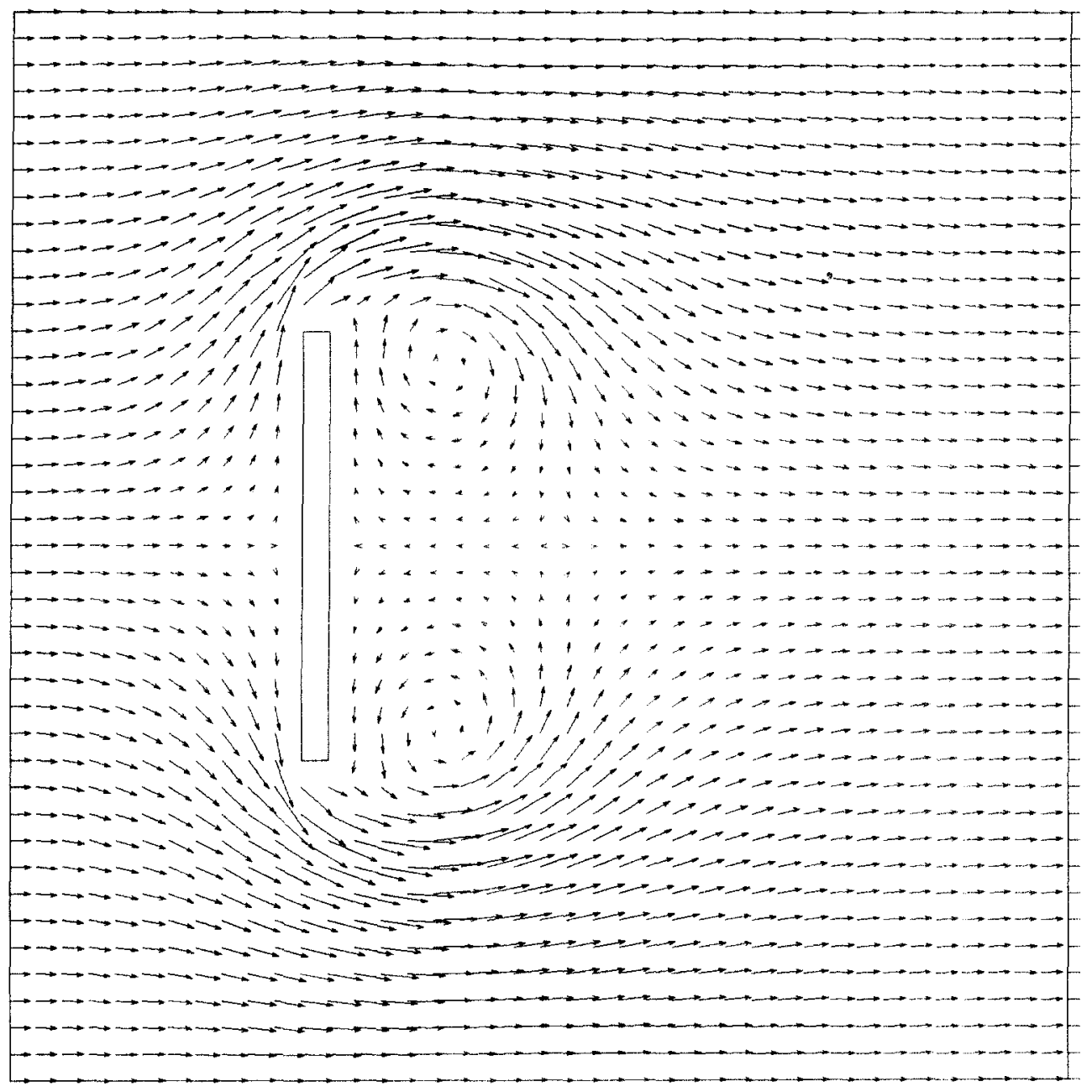

Fig. 16(a) 


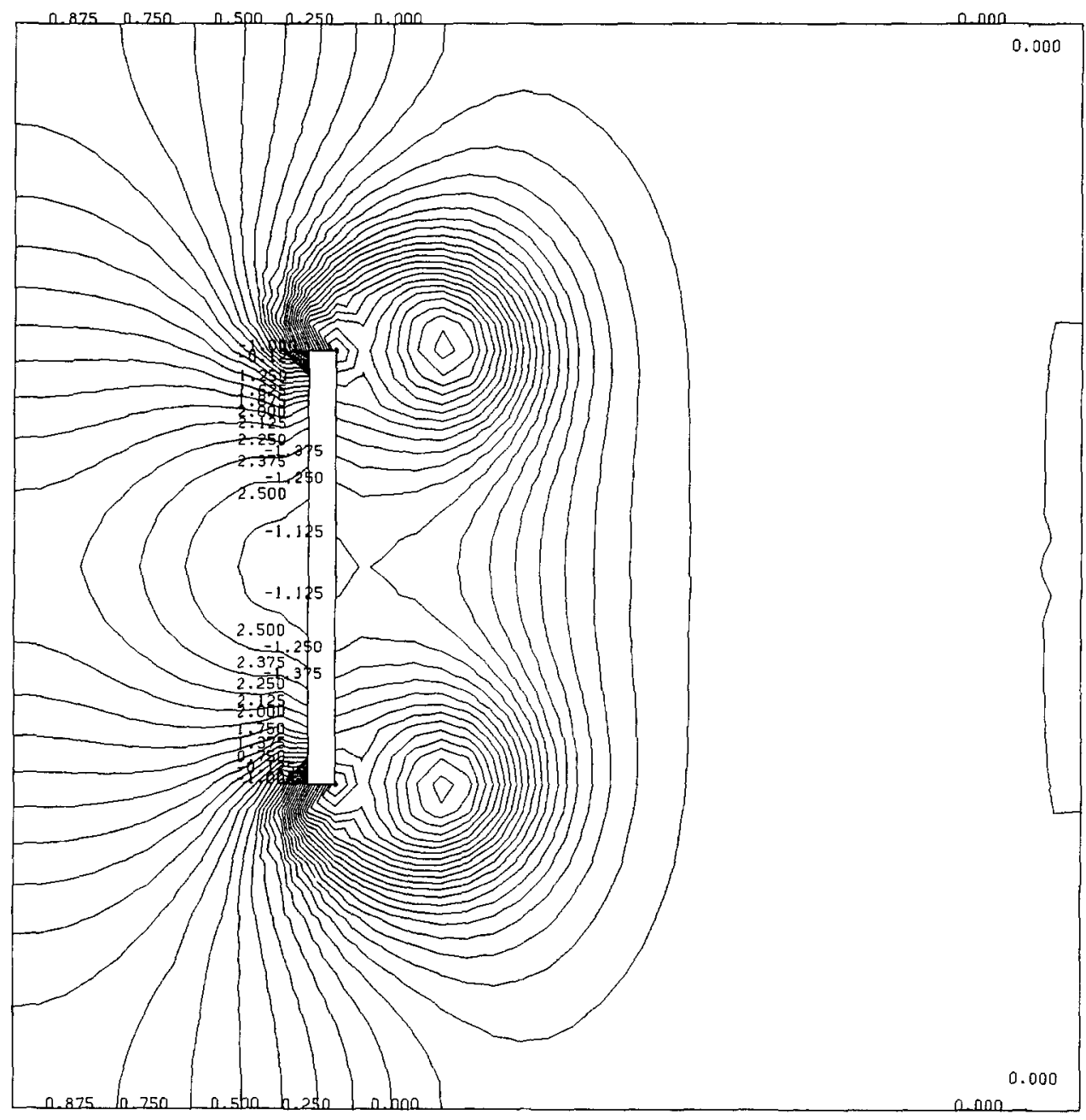

Fig. 16(b) 


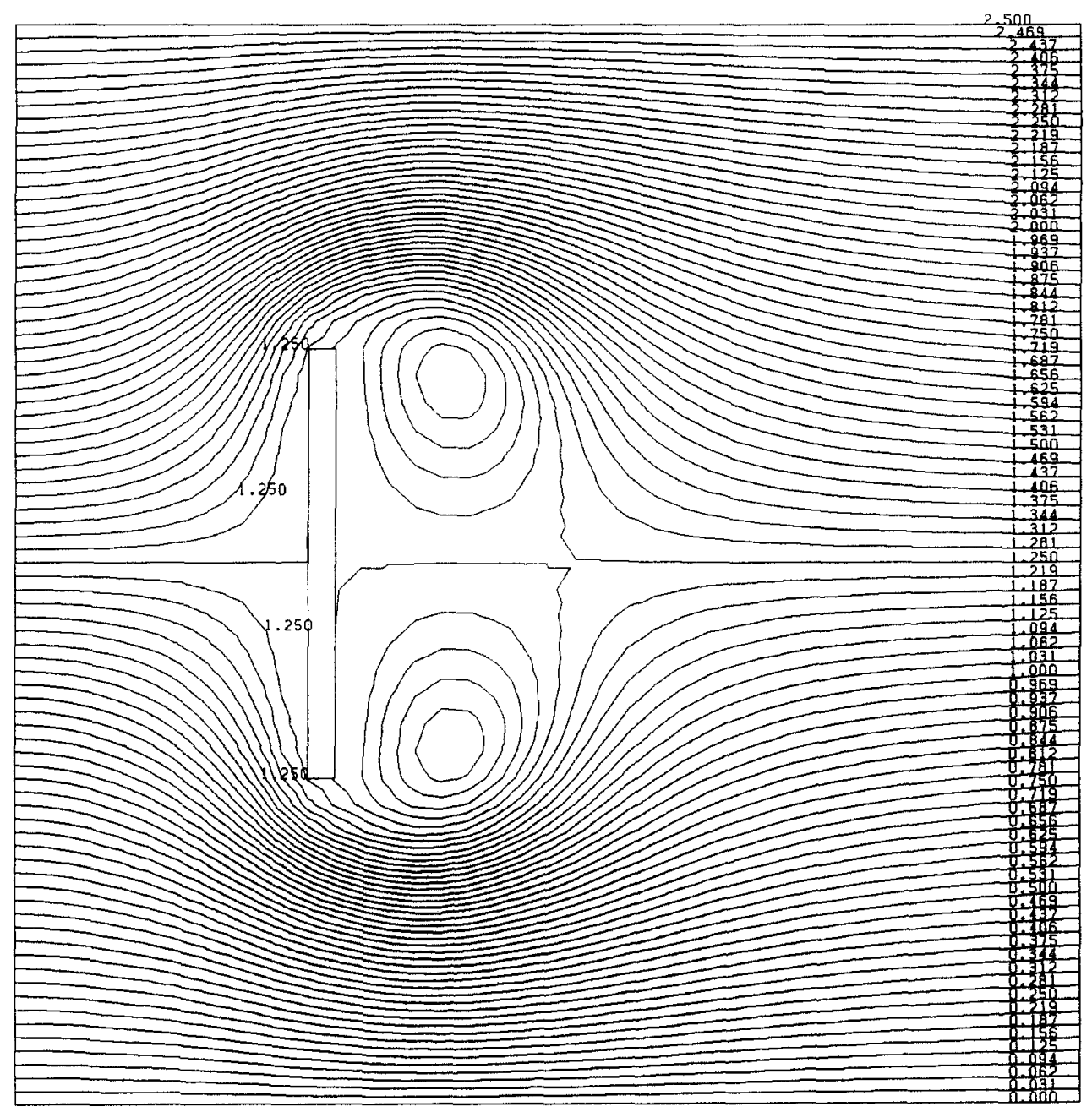

Fig. 16(c)

Fig. 16. Unsteady flow at $\operatorname{Re}=100, t=0.5$. (a) Velocity vectors; (b) pressure contours; (c) streamlines 


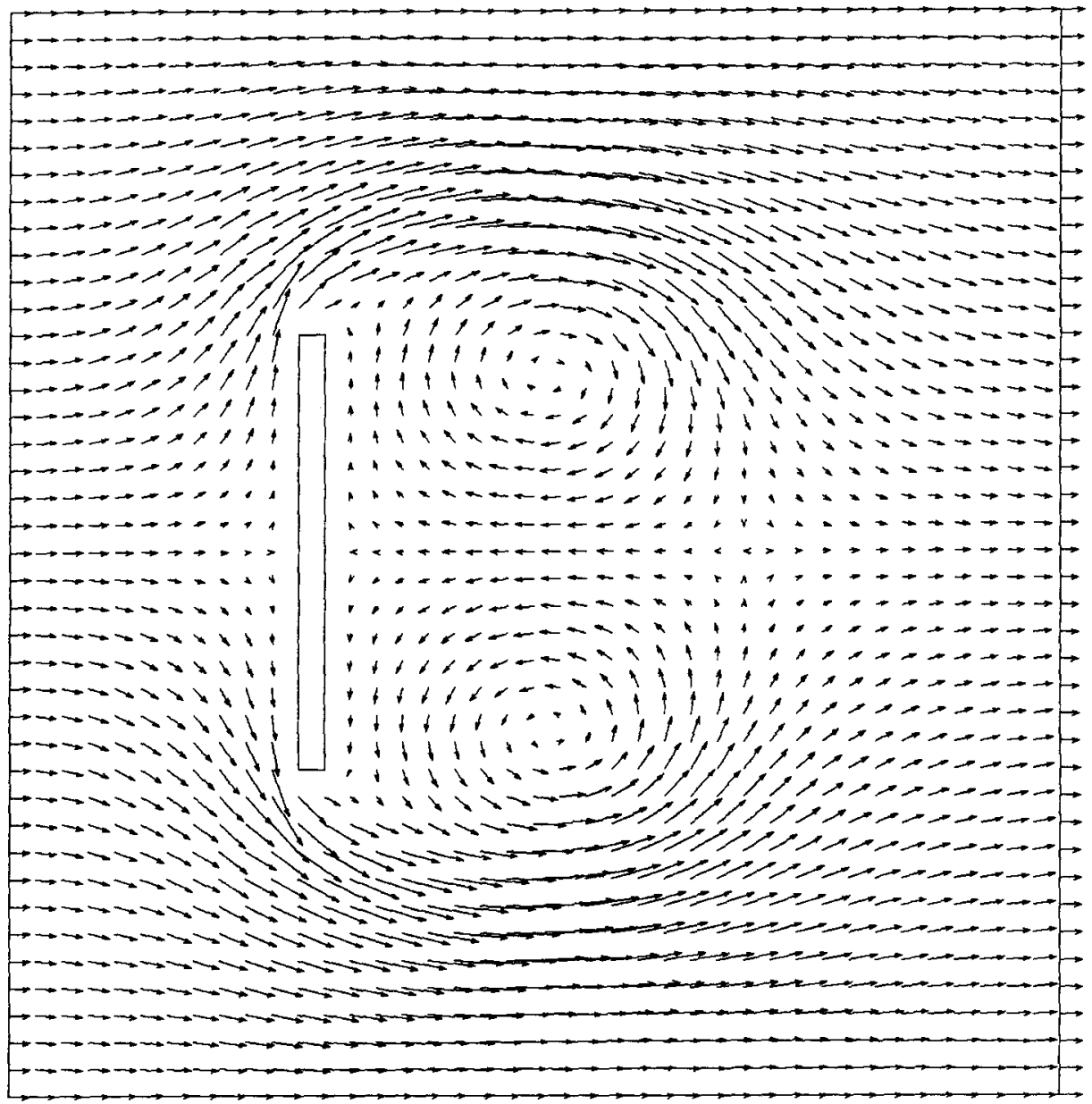

Fig. 17(a) 


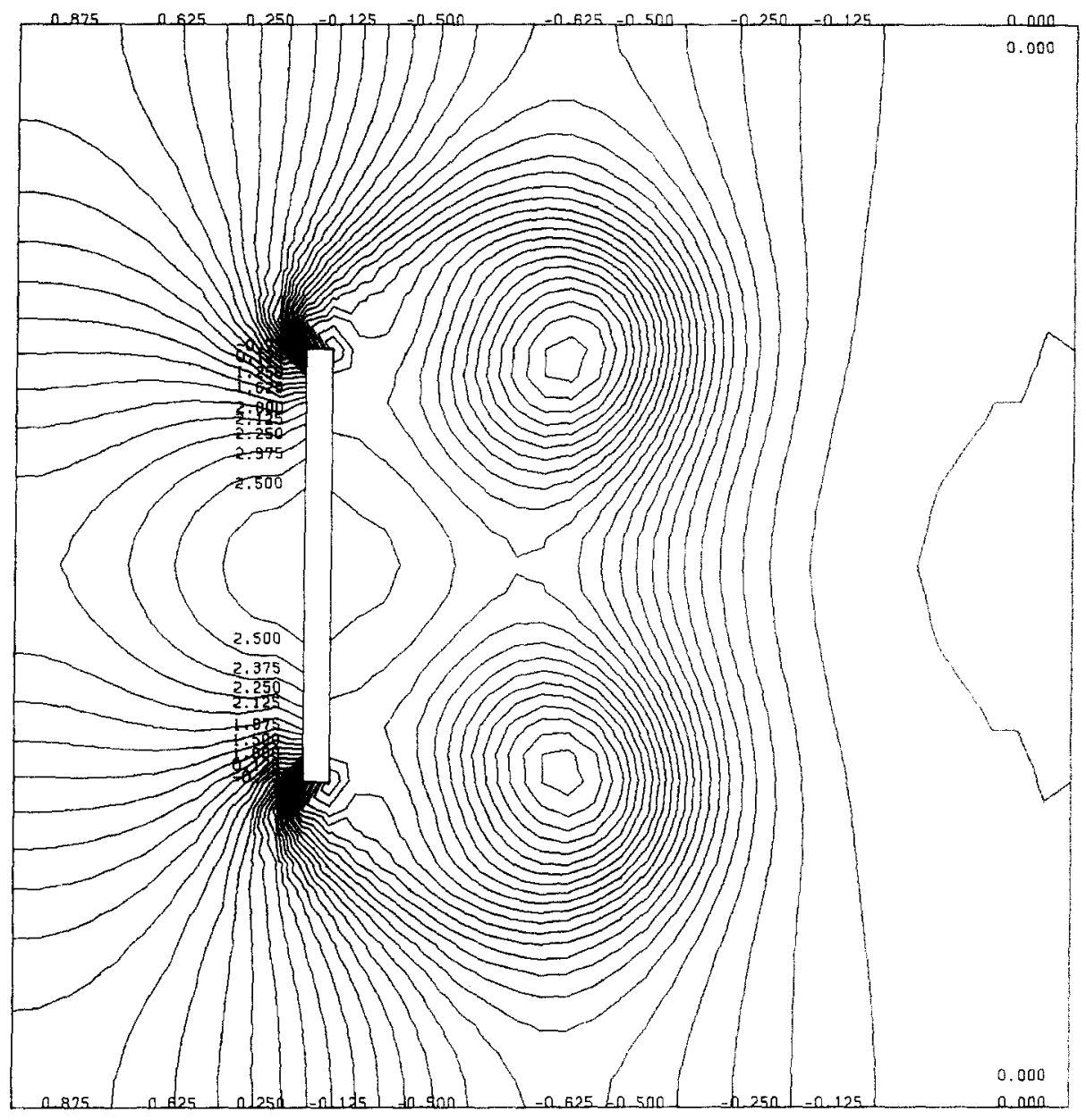

Fig. 17(b) 


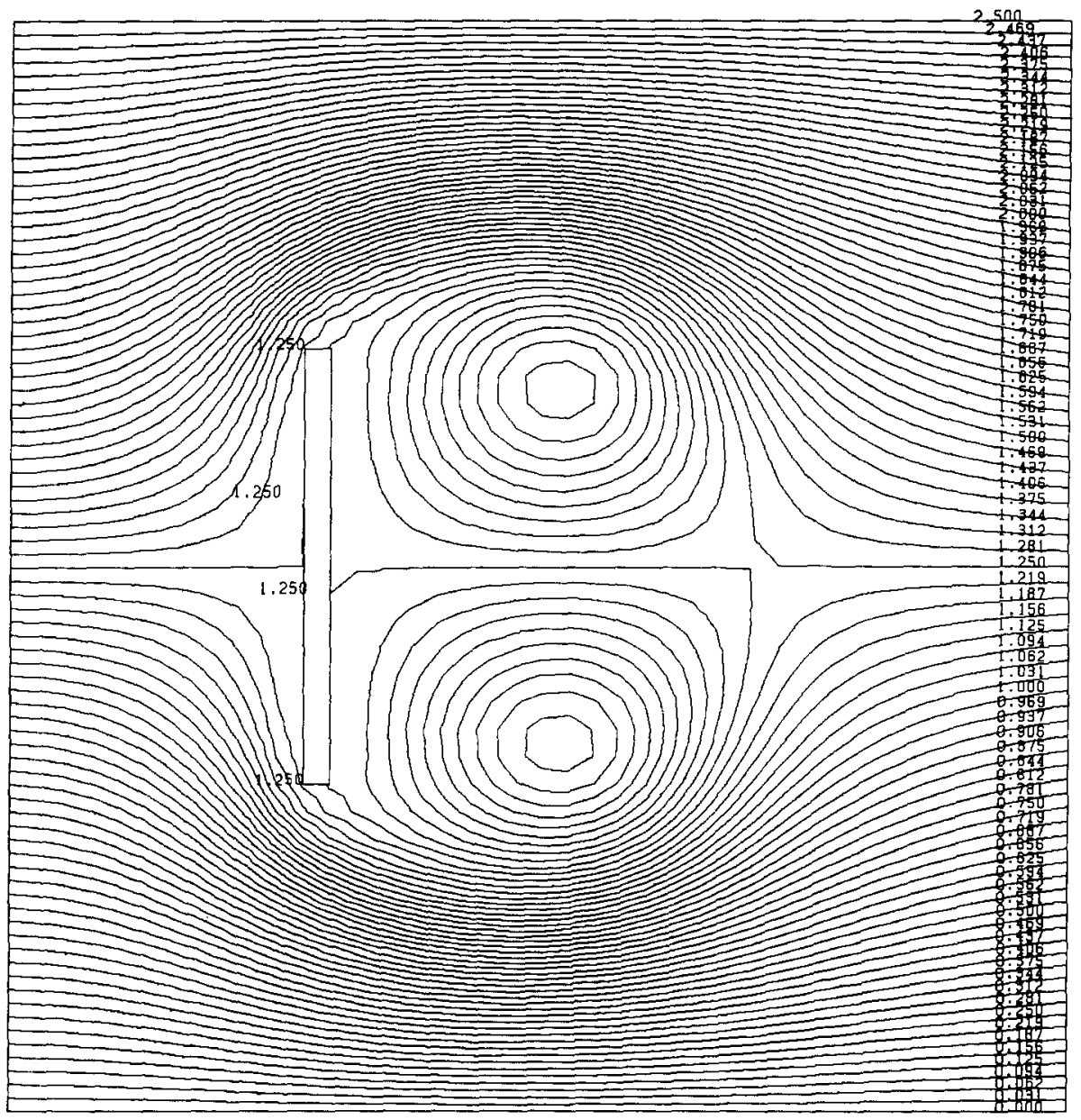

Fig. 17(c)

Fig. 17. Unsteady flow at $\operatorname{Re}=100, t=1.0$. (a) Velocity vectors; (b) pressure contours; (c) streamlines. 


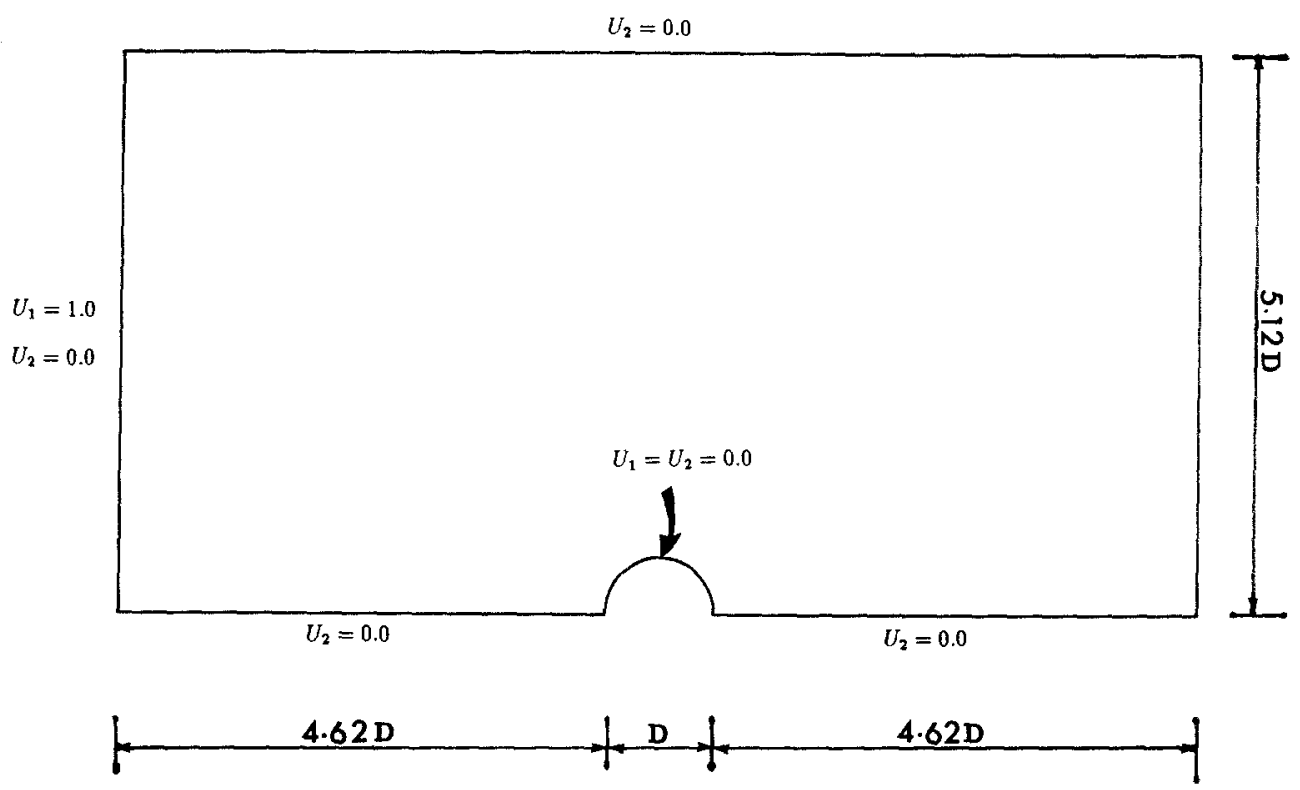

(a)

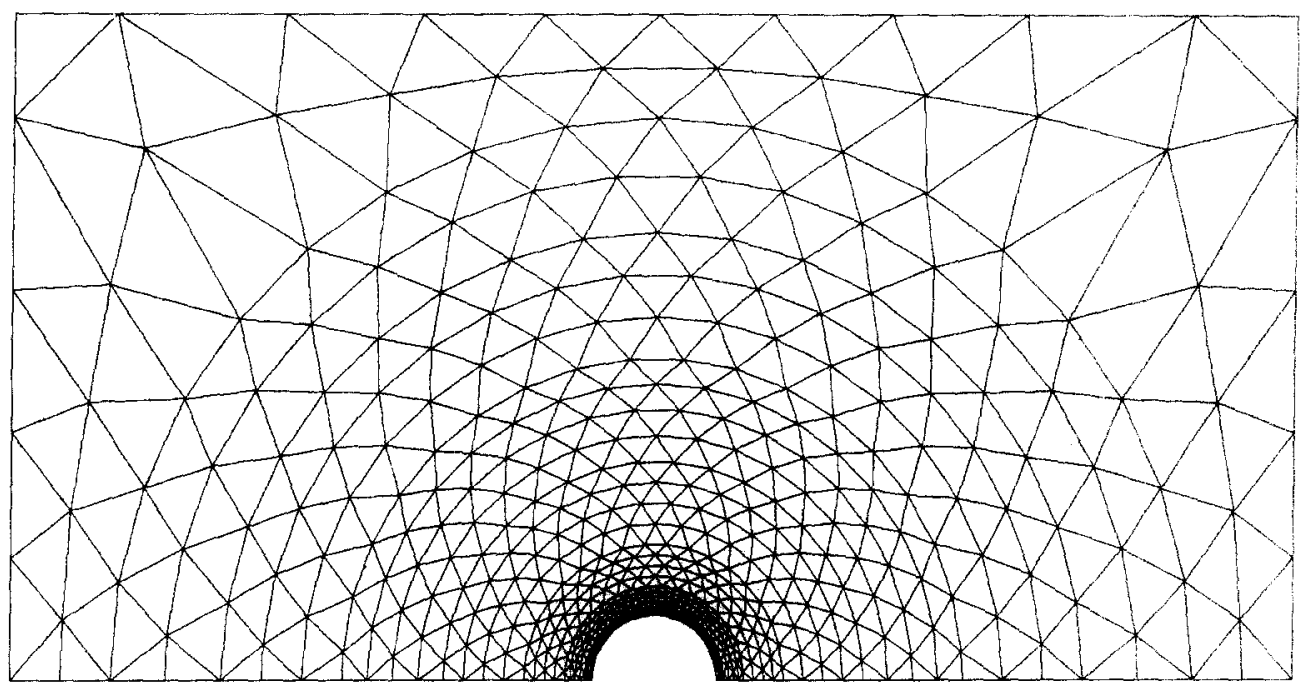

(b)

Fig. 18. Flow around a stationary sphere. (a) Problem description; (b) finite element mesh

on the horizontal boundaries. The S-shaped pattern observed for high Ra by Stevens [12] and Elder [13] are clearly seen from the isotherm lines. For $\mathrm{Ra} \geqslant 0.5 \times 10^{6}$ it is apparent that an isothermal core exists in the cavity with uniform temperature $\Theta=\frac{1}{2}$. This was postulated by Batchelor [14] for $\operatorname{Ra} \rightarrow \infty$.

It is to be expected that, for the present problem a symmetry exists in the sense that the field in the lower half of the cavity is a negative image of the field in the upper half provided the boundary condition on top and bottom of the cavity are the same. This requires value at midpoint equal to 0.5 . This value is satisfied exactly for all the cases considered.

7.6.2. Stream function. The streamlines in a square cavity for increasing values of Rayleigh number upto $10^{7}$ are shown in Fig. 23 for insulated boundary conditions. For $\mathrm{Ra}<10^{5}$ the flow is unicellular and clockwise and shows a circulation pattern up the hot wall and down the cold wall. For higher $\mathrm{Ra}=10^{5}$ the development of a boundary layer near the wall is clearly demonstrated and a second set of streamlines also with clockwise circulation represents the 


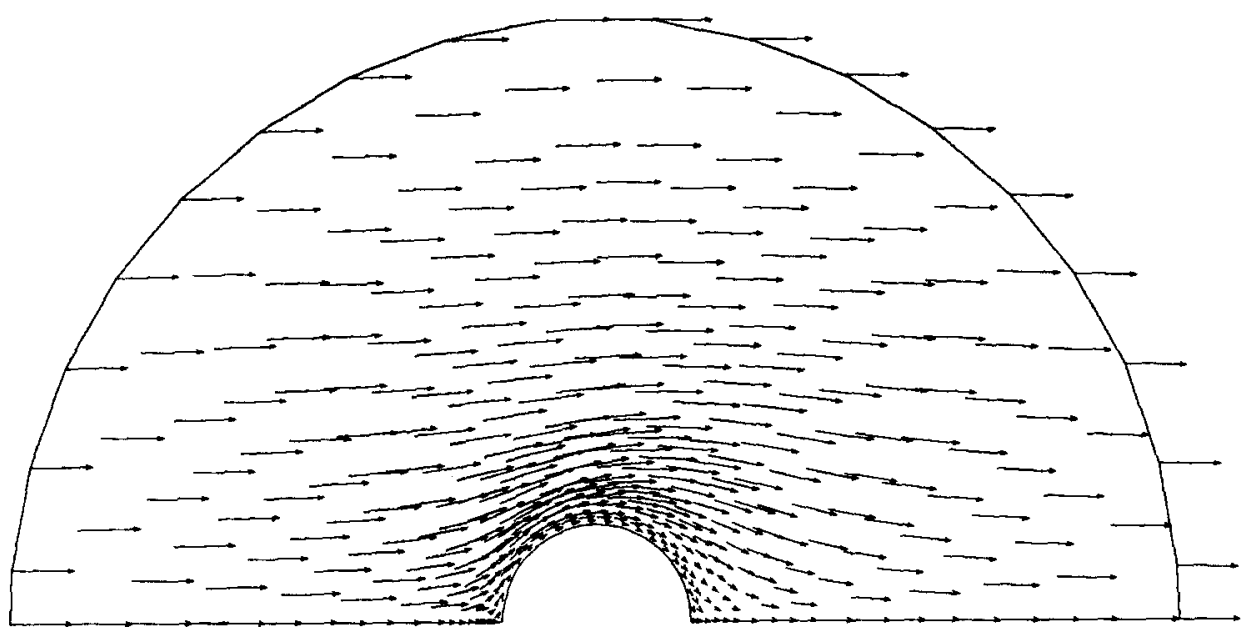

(a)

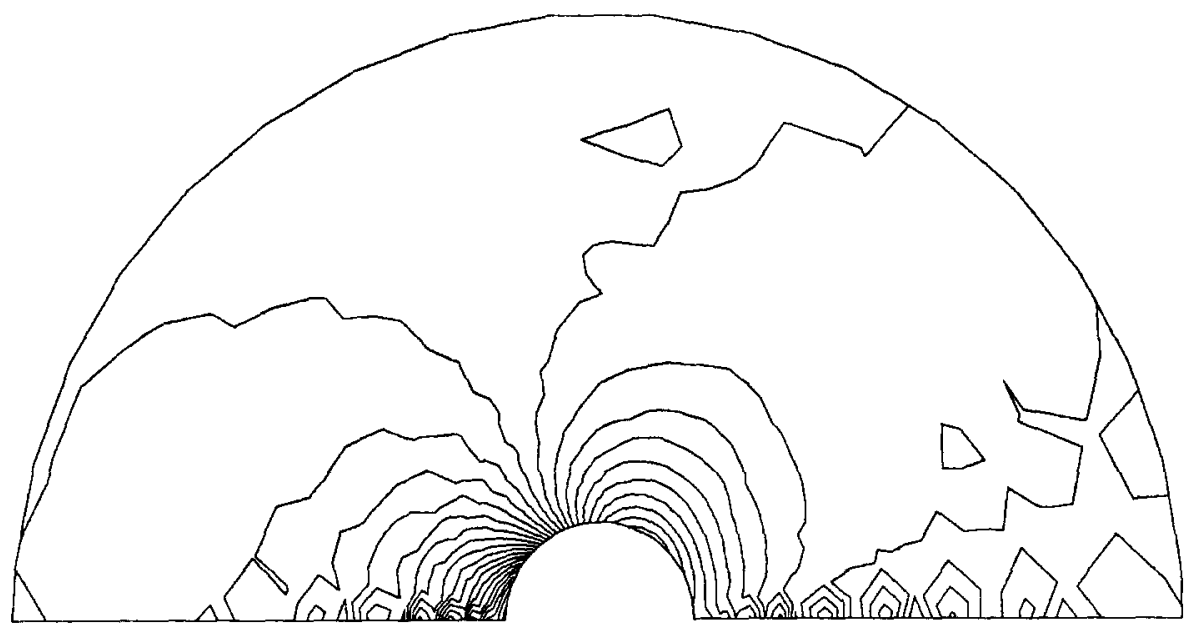

(b)

Fig. 19. Computed steady-state solution for flow around a stationary sphere at $\operatorname{Re}=10$. (a) Velocity vectors; (b) pressure contours.

\section{- D Present FEM}

- Analytical solution
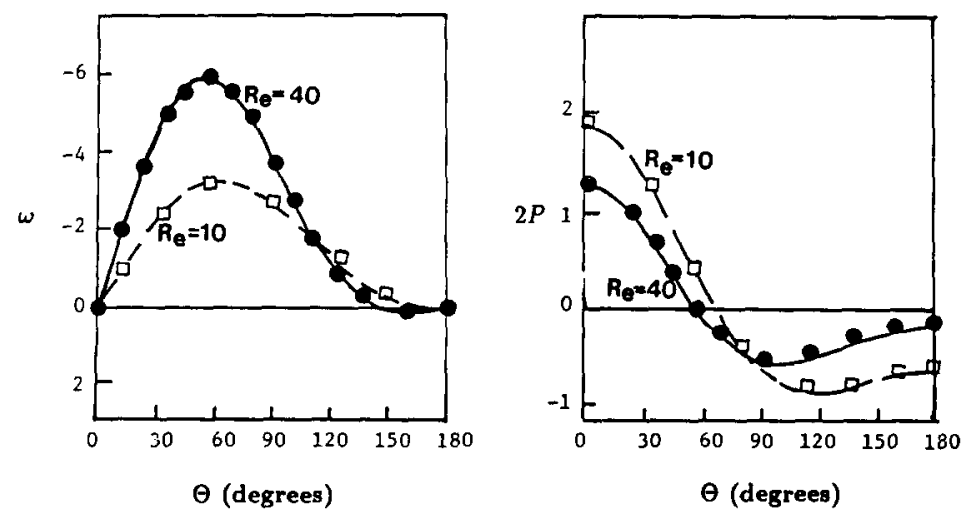

Fig. 20. Flow around a stationary sphere. Vorticity and pressure distribution over the surface of the sphere for $\operatorname{Re}=10$ and 40 . 


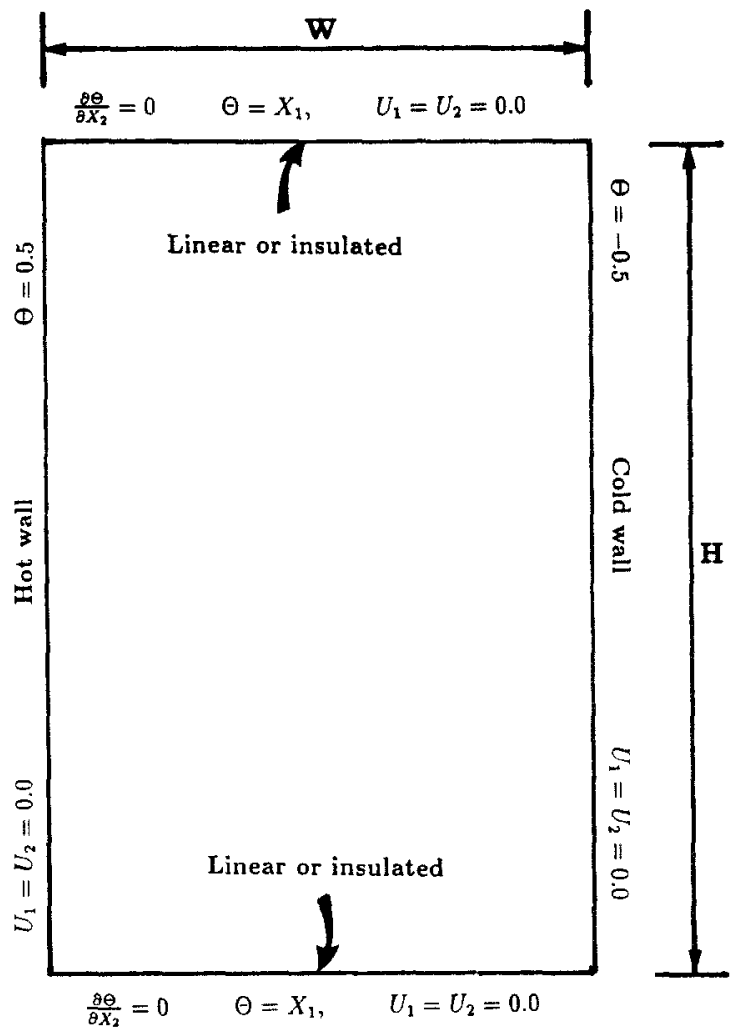

Fig. 21. The cavity geometry and boundary conditions for natural convection flows

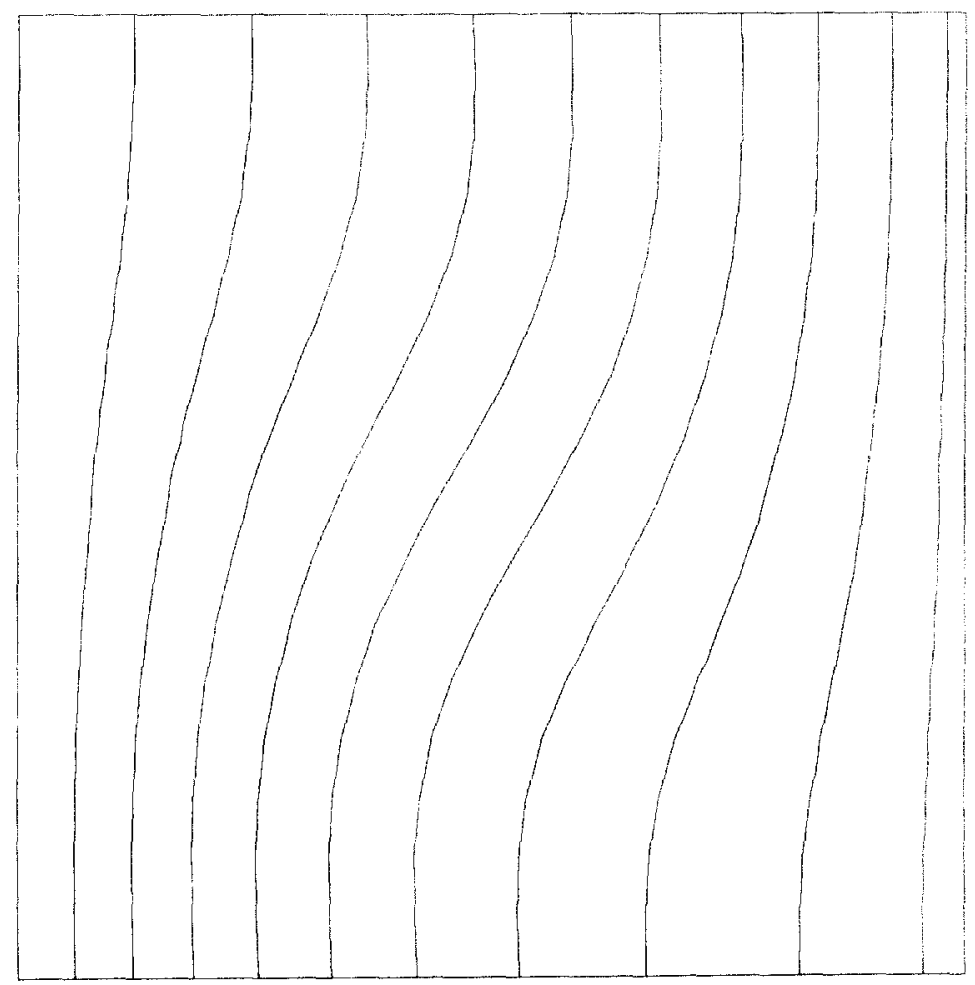

Fig. 22(a) 


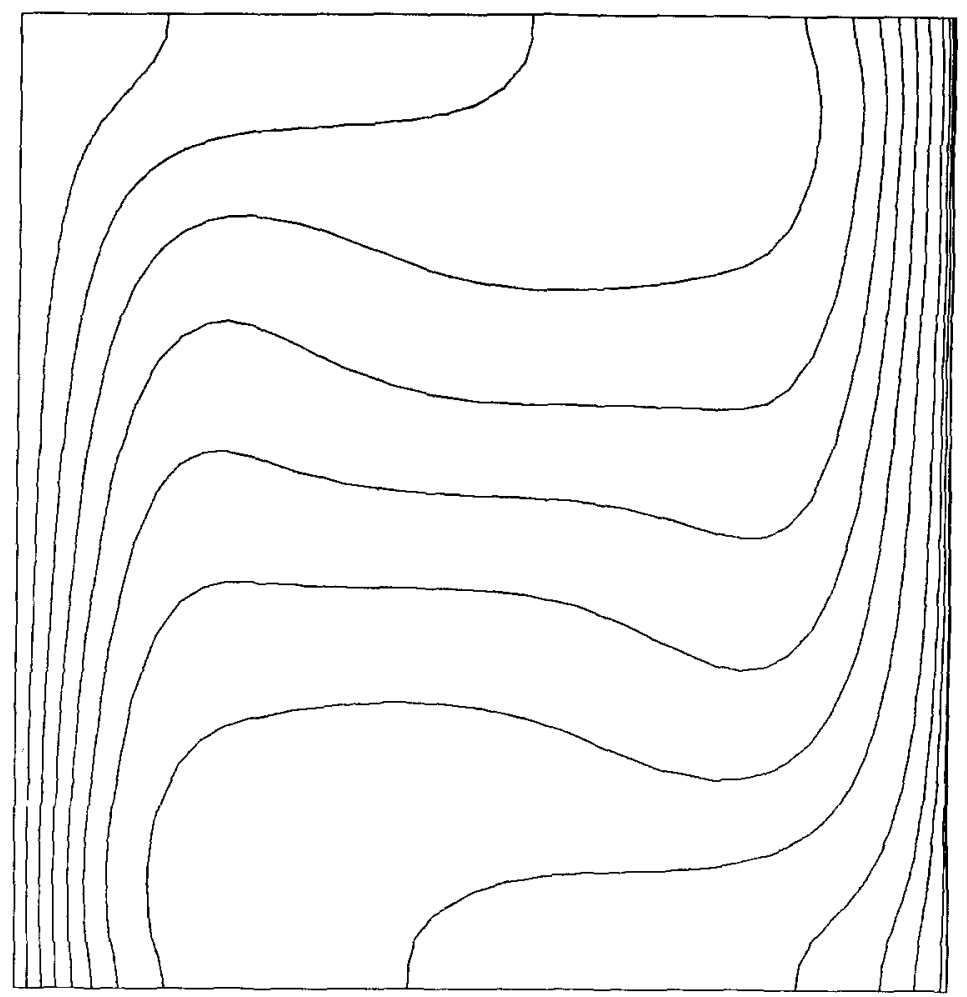

Fig. 22(b)

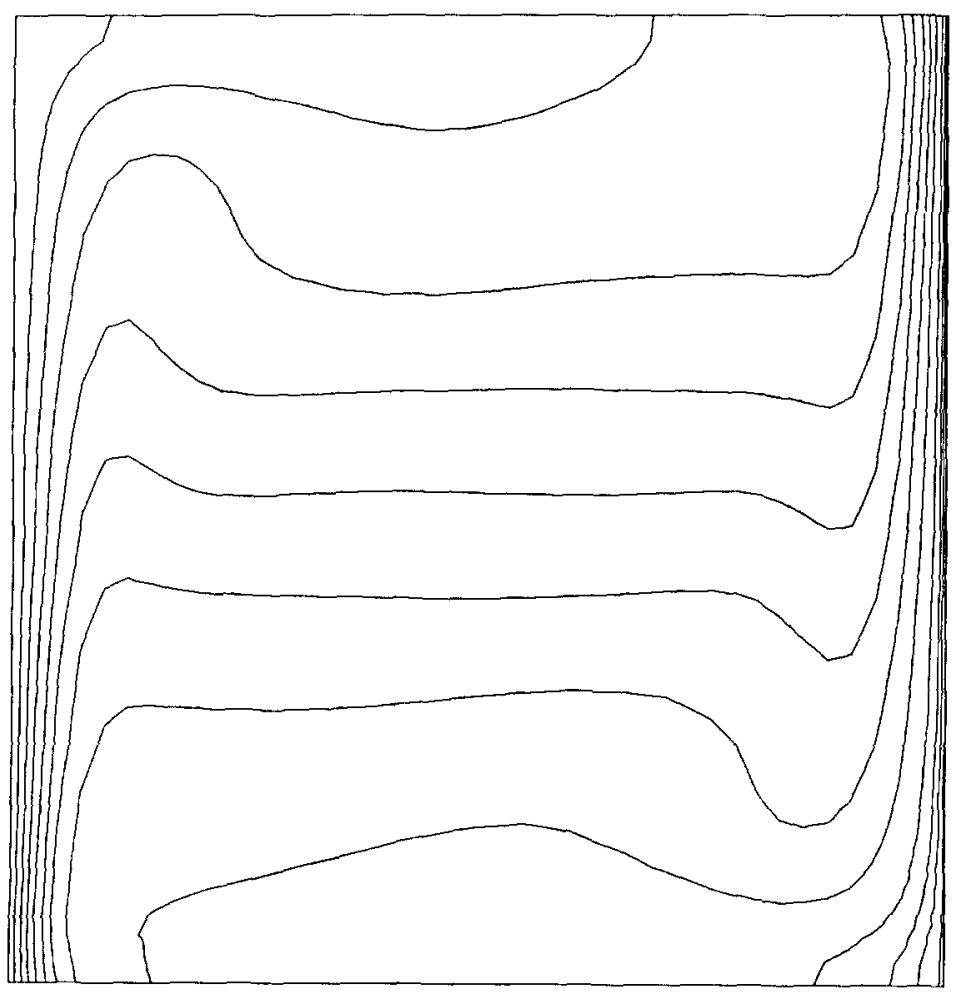

Fig. 22(c) 


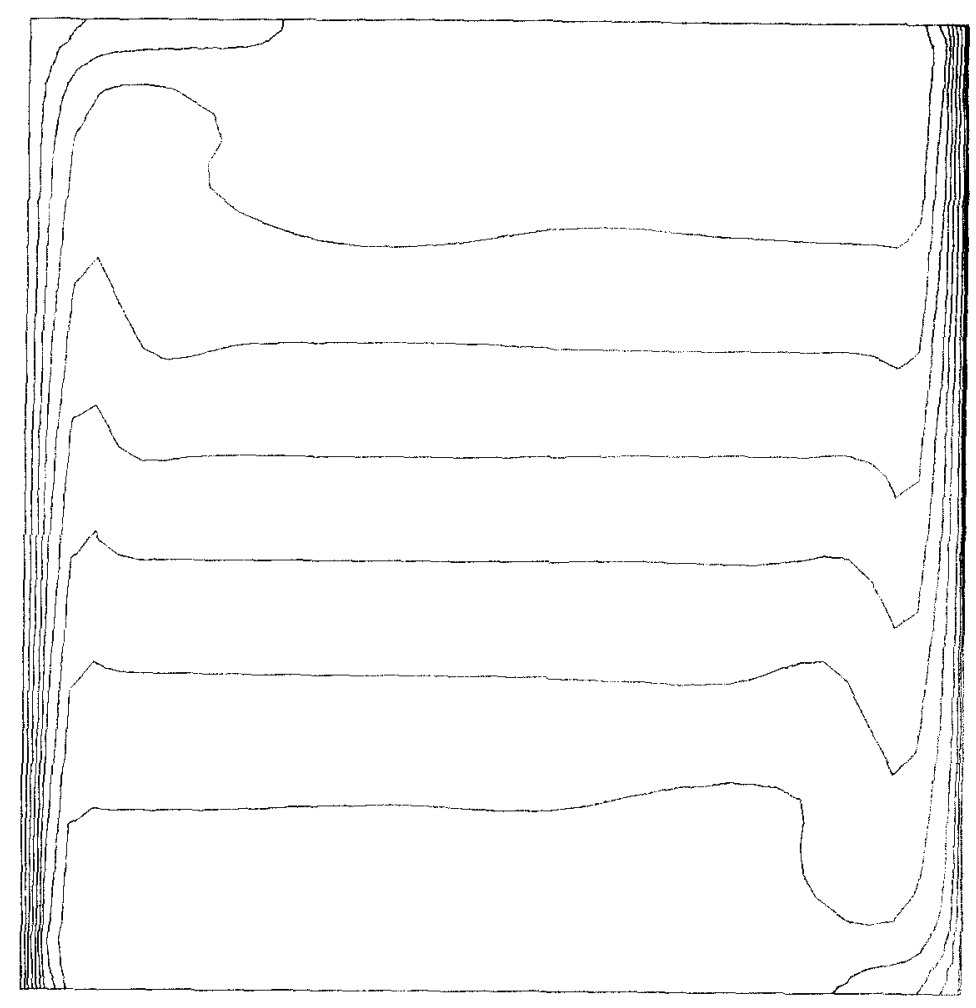

Fig. 22(d) Fig, 22. Steady-state isotherm lines for square cavity. (a) $\mathrm{Ra}=10^{3}$; (b) $\mathrm{Ra}=10^{5}$; (c) $\mathrm{Ra}=10^{6}$;
(d) $\mathrm{Ra}=10^{7}$.

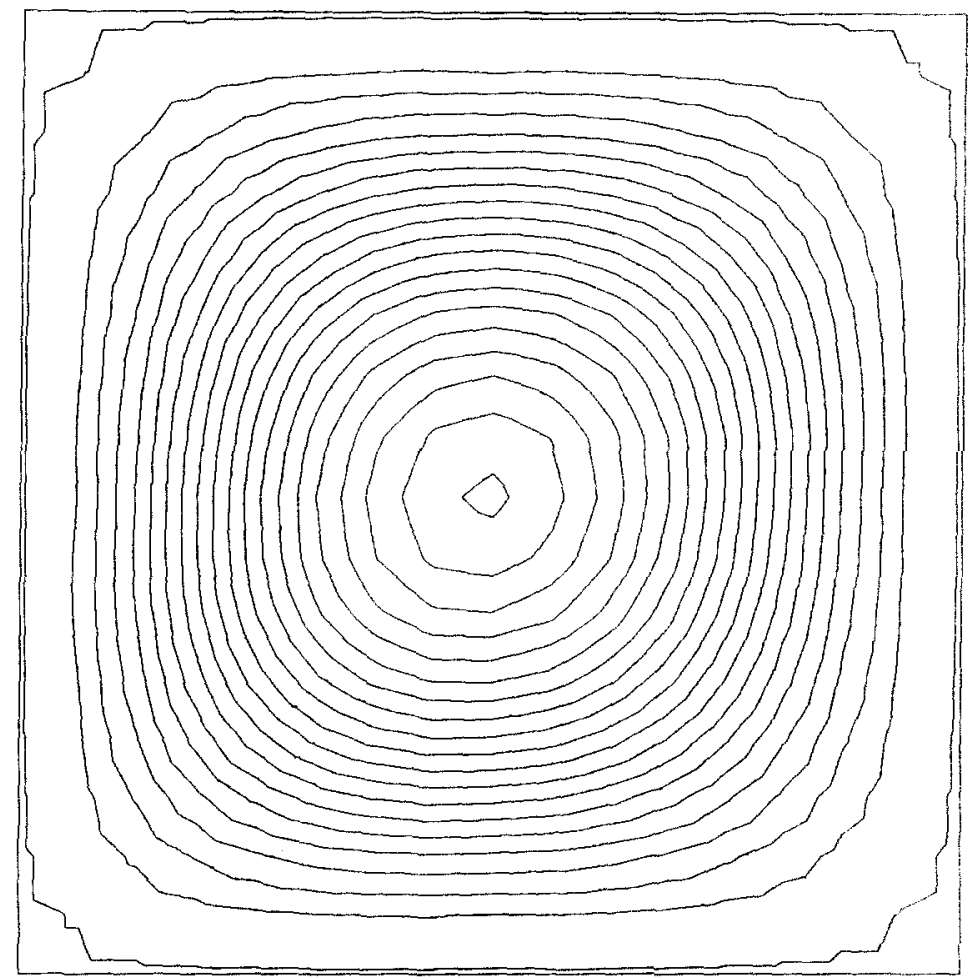

Fig. 23(a) 


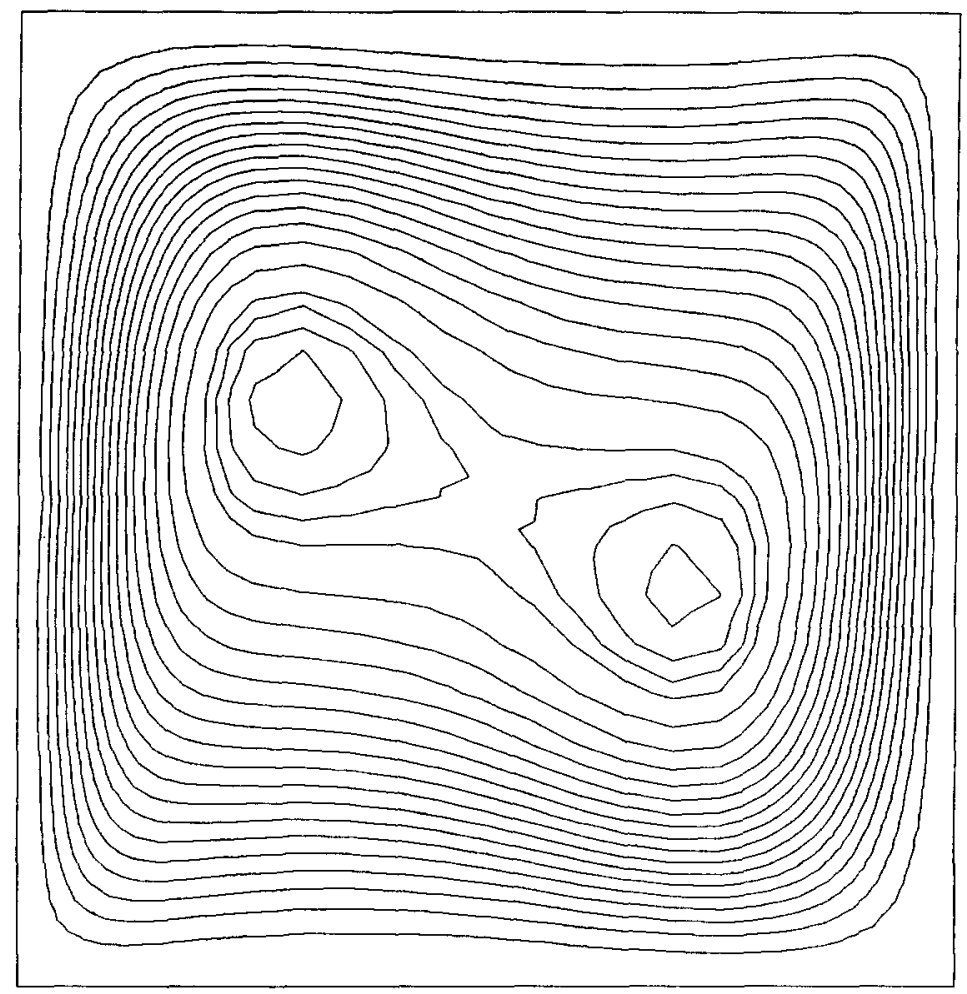

Fig. 23(b)

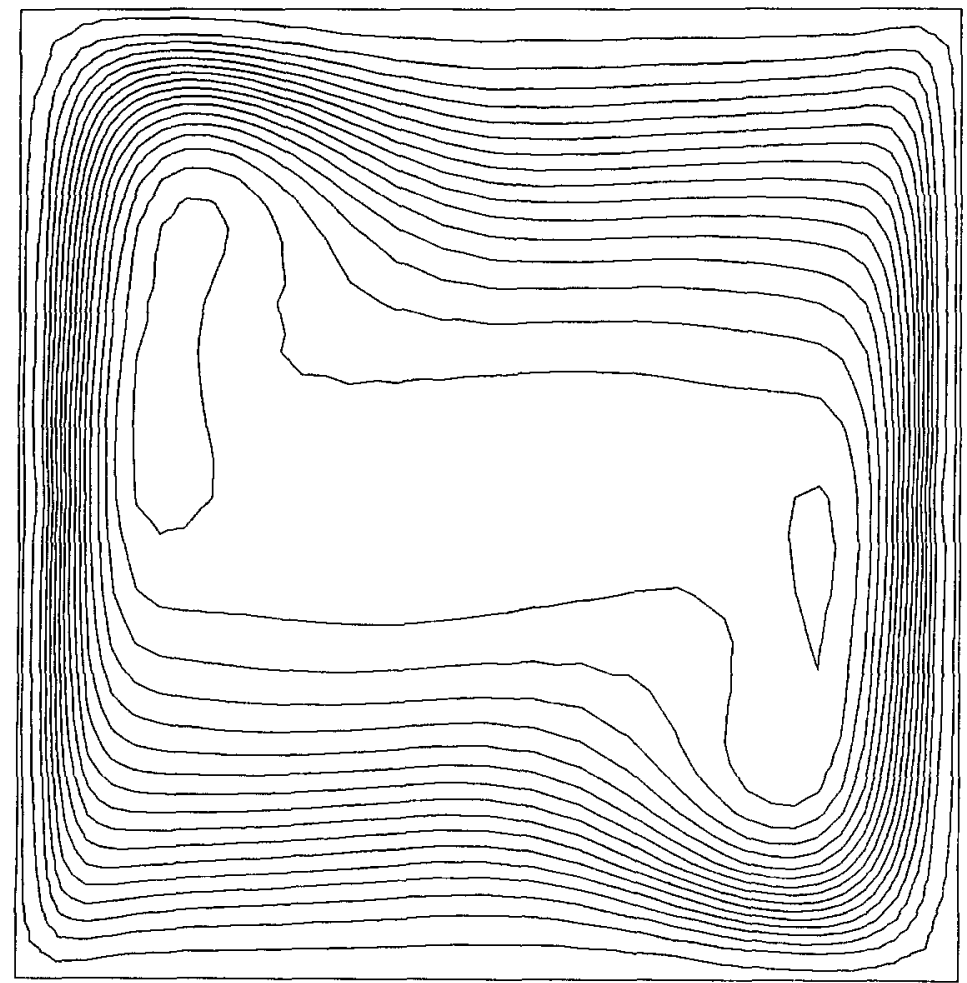

Fig. 23(c) 


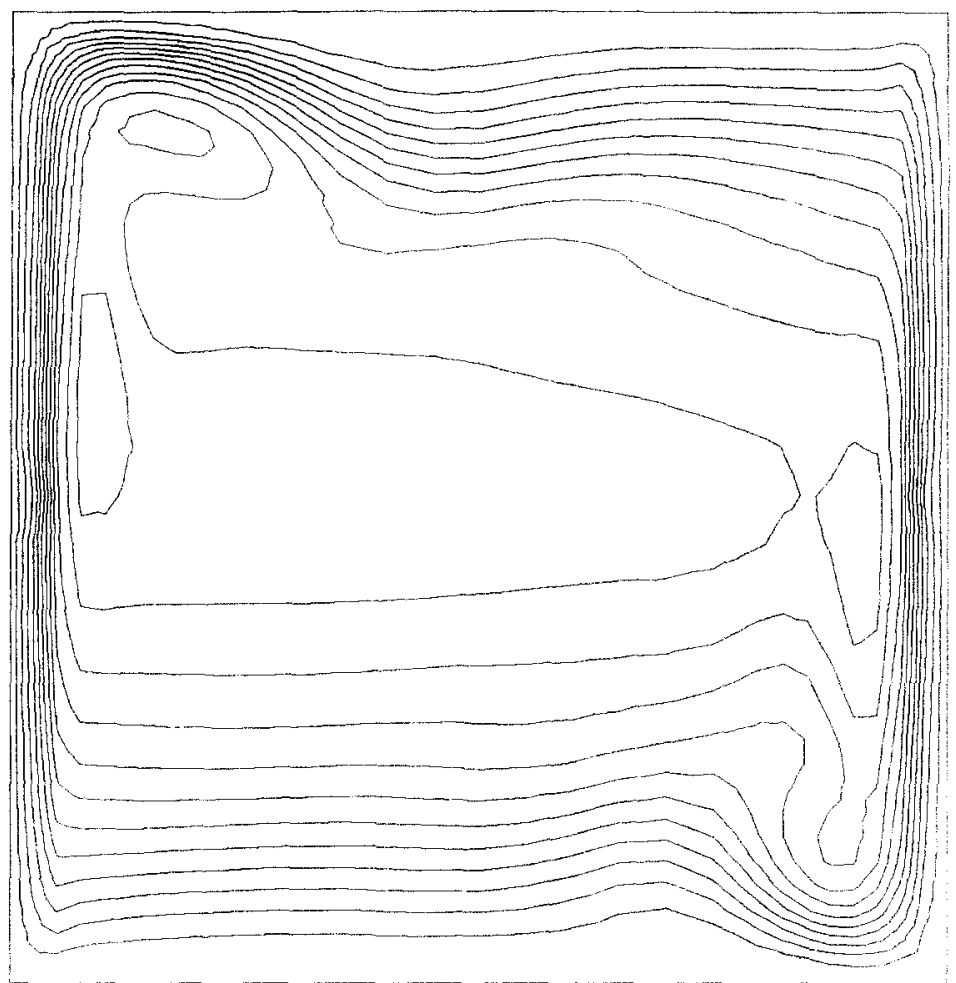

Fig. 23(d)

Fig. 23. Steady-state streamlines for square cavity. (a) $\mathrm{Ra}=10^{3}$; (b) $\mathrm{Ra}=10^{5}$; (c) $\mathrm{Ra}=10^{6}$; (d) $\mathrm{Ra}=10^{7}$.

secondary flow occurs in the central region of the cavity. This is in agreement with Elder's cats-eye pattern [13] and the results of DE Vahl Davis [15] and Rubel and Landis [16].

7.6.3. Comparison of stream function and average Nusselt number with previous results. Figure 24 shows the values of stream function at the cavity mid-point as a function of Rayleigh number for insulated boundary condition. These are compared with previous other numerical techniques. The agreement between the present finite element solution and the finite difference solution is excellent in all respects.

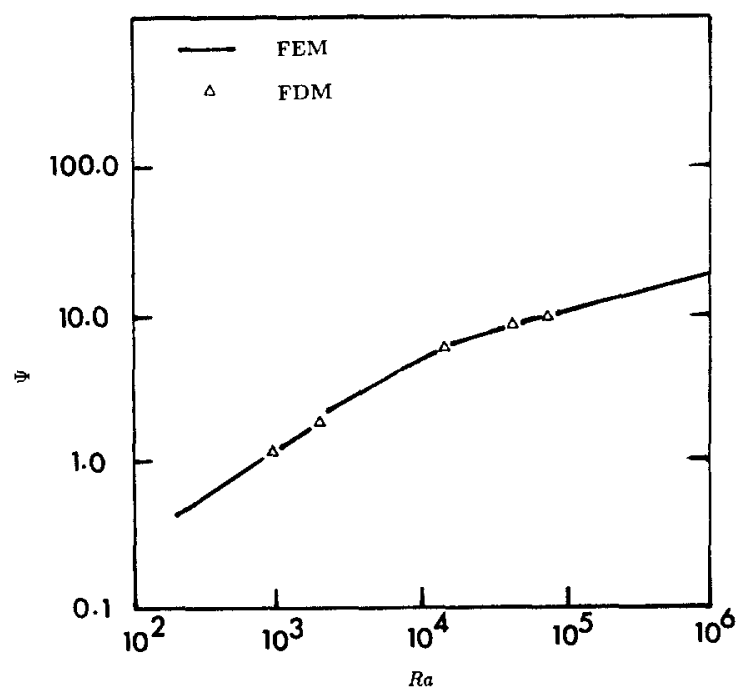

Fig. 24. Stream function at the cavity mid-point. 


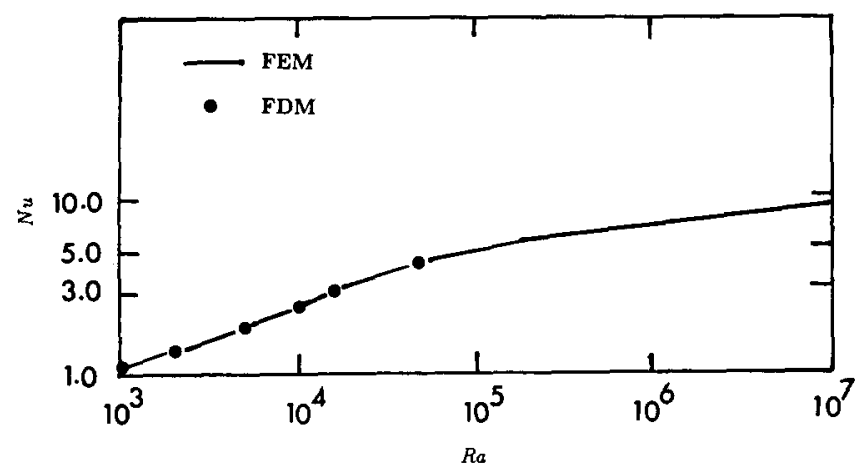

Fig. 25. Averge Nusselt number for square cavity.

Figure 25 shows the average Nusselt number calculated by Gaussian integration as a function of the Rayleigh number. These results agree well with published finite difference solutions for insulated boundary conditions.

7.6.4. The effect of aspect ratio. Figures 26-28 show the effect of aspect ratio (height to width of the enclosure) on the temperature and flow fields. Figure 26 shows the isotherms and streamlines for a rectangular enclosure of aspect ratio 3. The Rayleigh number and Prandtl number are the same as those used by Hellums and Churchill [17]: $\mathrm{Ra}=1.466 \times 10^{4}, \operatorname{Pr}=0.733$. The present results agree qualitatively with those of Hellums and Churchill. Figure 27 shows similar results for a rectangular enclosure of aspect ratio 1.83 and with linear temperature distribution on the horizontal walls as indicated in the figure $(\mathrm{Ra}=8,200, \mathrm{Pr}=2,450)$. This example is the same as that considered by Shekely and Todd [18] who have compared experimental and finite difference solutions. The plotted values of the steady state isotherms seem to agree well with those of Szekely and Todd.
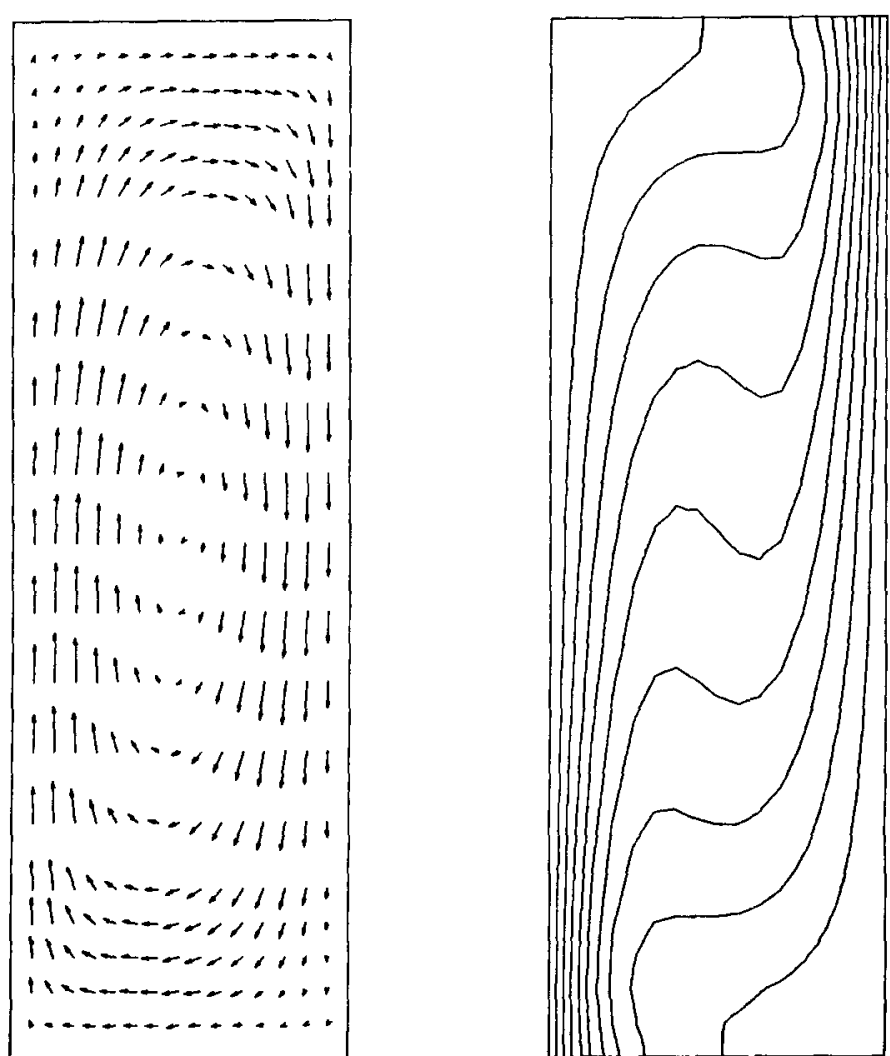

Fig. 26. Steady-state isotherms and streamlines for aspect ratio $3.0: \mathrm{Ra}=1.466 \times 10^{4}, \operatorname{Pr}=0.733$. 

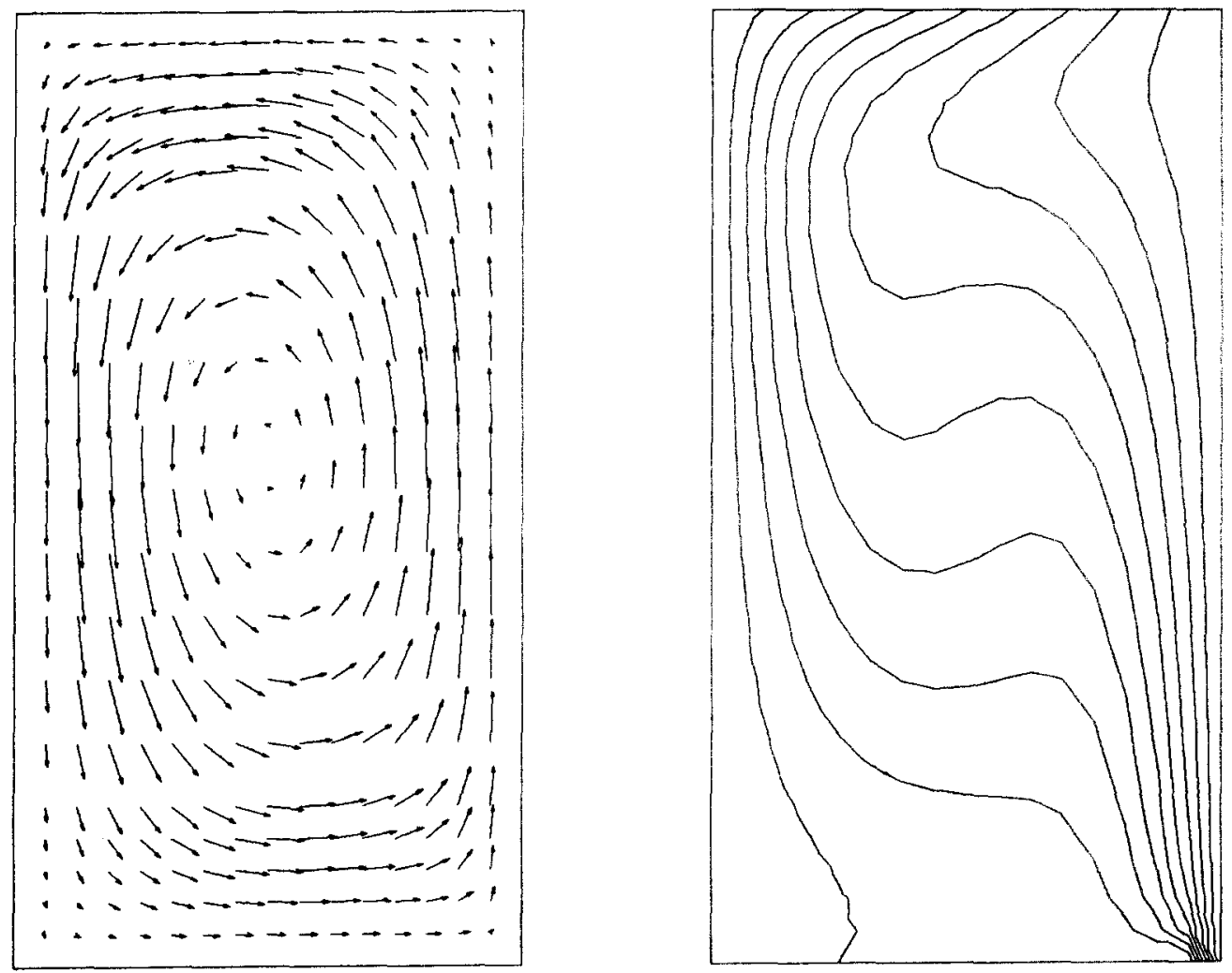

Fig. 27. Steady-state isotherms and streamlines for aspect ratio 1.83: $\mathrm{Ra}=8,200, \operatorname{Pr}=2,450$.
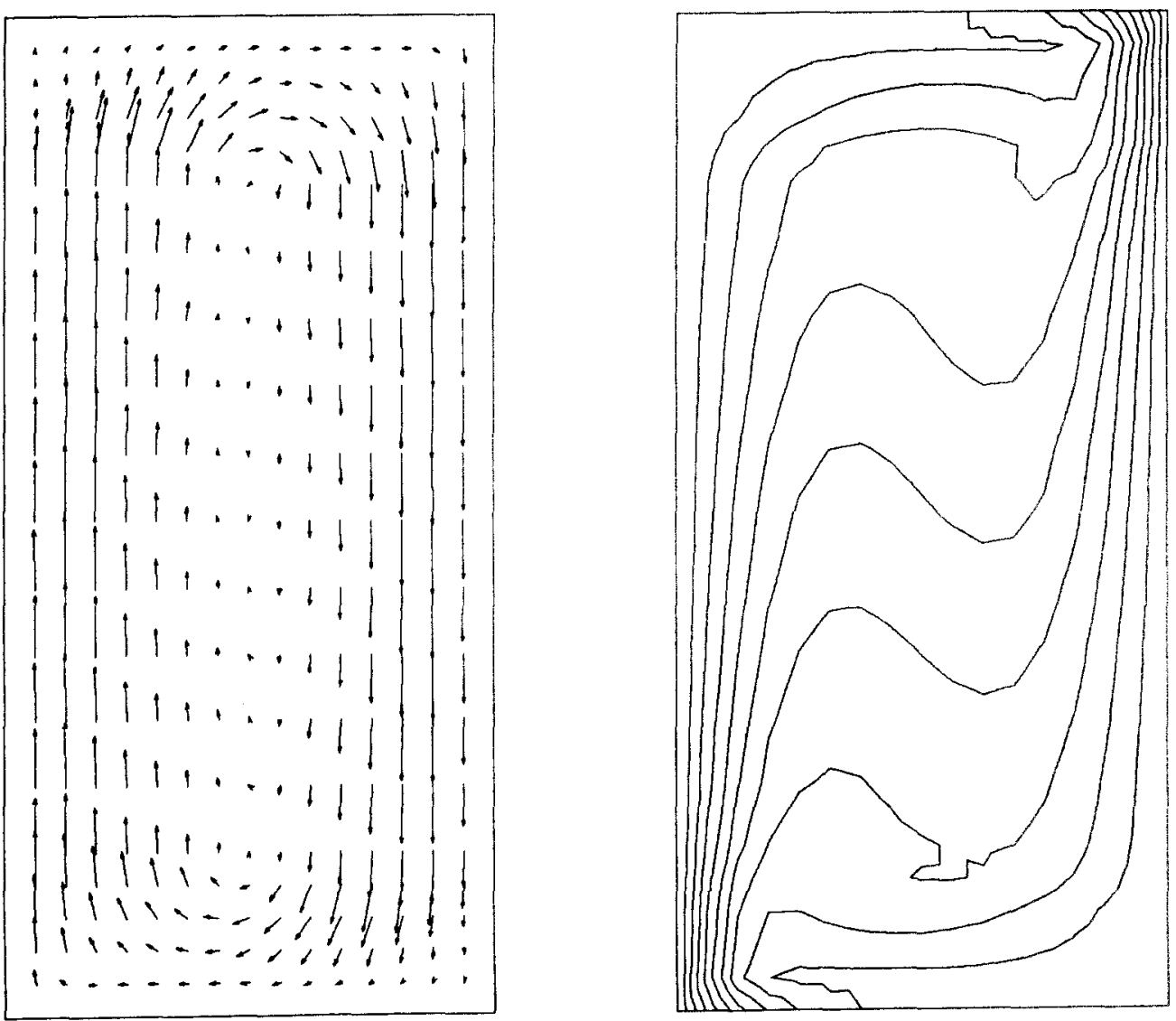

Fig. 28. Steady-state isotherms and streamlines for aspect ratio 10.0: $\mathbf{R a}=10^{5}, \mathrm{Pr}=0.71$. 
Finite element solution for advection and natural convection flows

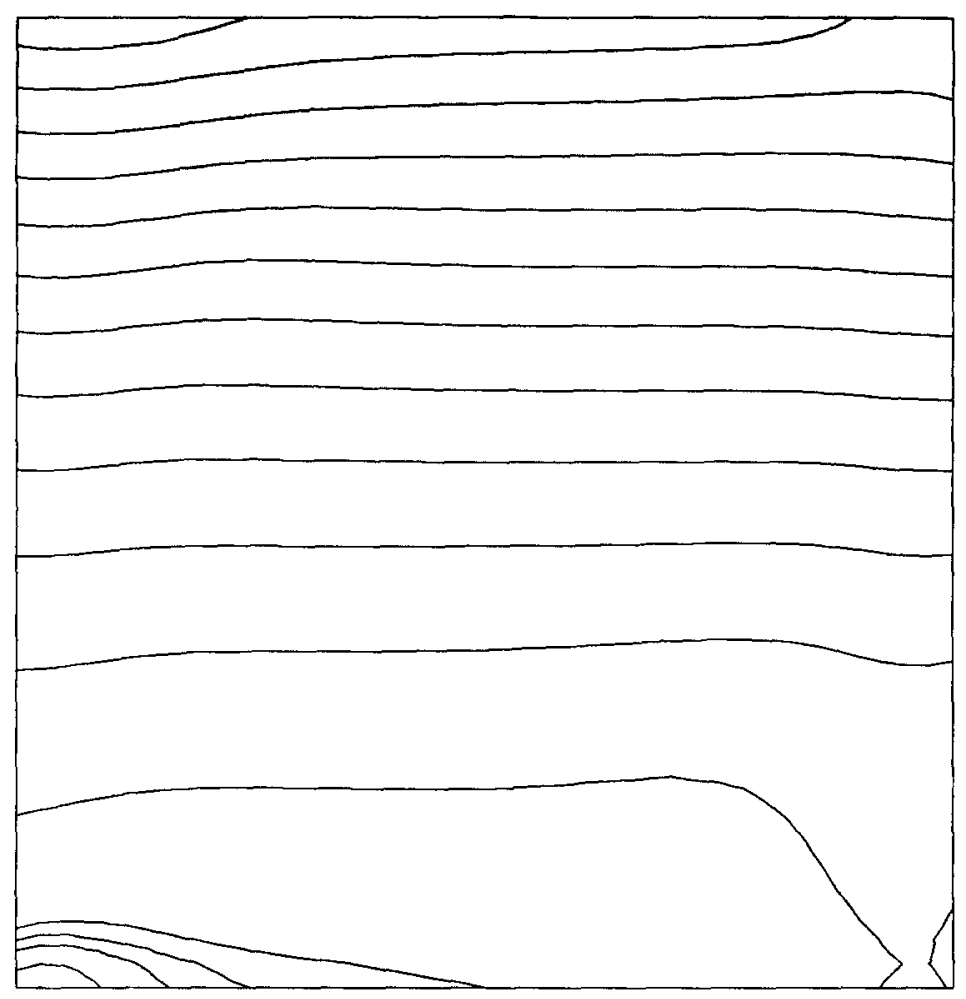

(a)

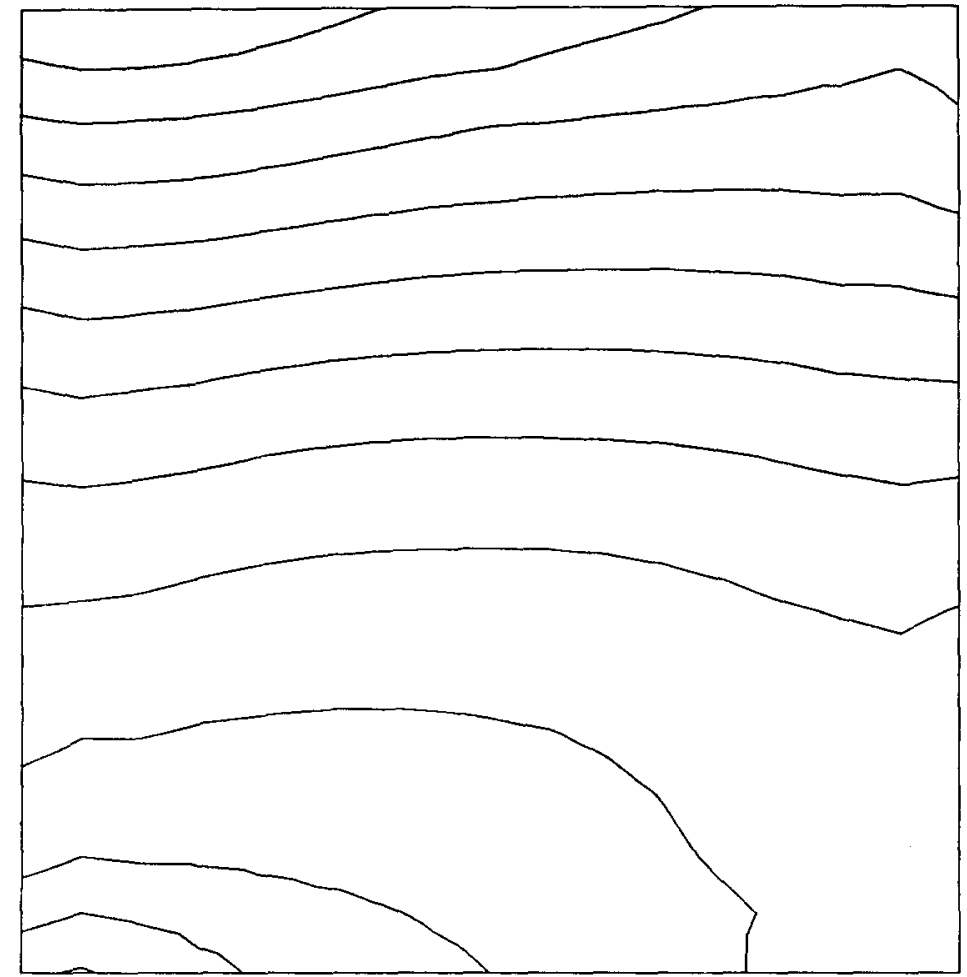

(b)

Fig. 29. Steady-state pressure distribution for a square cavity. (a) $\mathrm{Ra}=10^{5}$; (b) $\mathrm{Ra}=10^{7}$, 
Finally, Fig. 28 shows the results for an aspect ratio of $10, \mathrm{Ra}=10^{5}$ and $\operatorname{Pr}=0.71$. In general it was observed that for larger aspect ratios the numerical solutions converged slower.

7.6.5. Pressure distribution. Figure 29 shows the steady state contour plots of pressure at $\mathrm{Ra}=10^{5}$ and $10^{7}$. It is expected that the pressure field is influenced by the cavity corners. For high $\mathrm{Ra} \geqslant 10^{5}$ the pressure becomes more uniform across the cavity except near the corners. The present results are found to be in good agreement with those obtained in Ref. [19] by means of a finite element method. These pressure contours were included to demonstrate that equal order velocity-pressure solution does not generate spurious pressure modes.

\section{CONCLUDING REMARKS}

In this paper, a new equal order velocity-pressure finite element model has been presented for advection and natural convection flows. The solution is based on Galerkin method of weighted residuals using $U_{i}, P$ and $\Theta$ as the main dependent variables. The required distribution of the stream function is also obtained using the finite element method. The results presented here illustrate that the method does not yield any spurious pressure modes for the example problems treated. In addition to the examples presented here, the author has successfully applied the method to a number of other cases. In all cases, no spurious pressure modes are produced. The overall iterative solution methods used in this work provide considerable savings in execution times and storage requirements, compared to other finite element methods.

Acknowledgements - The author would like to thank Professor M. Kawahara of Chuo University for his interest, encouragement, and many helpful discussions. The computation has been carried out using HITAC M680H computer of the University of Tokyo. The use of this computer facility is also gratefully acknowledged.

\section{REFERENCES}

1. F. H. Harlow and J. E. Welch, Numerical calculation of time-dependent viscous incompressible flow of fluid with free surface. Phys. Fluids 8, 2182-2189 (1965).

2. A. J. Chorin, Numerical solution of the Navier-Stokes equations. Math. Comput. 22, 745-762 (1968).

3. J. Kim and P. Moin, Application of a fractional-step method to incompressible Navier-Stokes equations. J. Comput. Phys. 59, 308-323 (1985)

4. C. T. Yang and S. N. Atluri, An assumed deviatoric stress pressure velocity mixed finite element method for unsteady, convective, incompressible viscous flow. Part I: Theoretical development. Int. J. Num. Meth. Fluids 3, $377-398$ (1983).

5. C. T. Yang and S. N. Atluri, An assumed deviatoric stress pressure velocity mixed finite element method for unsteady, convective, incompressible viscous flow. Part II: computational studies. Int. J. Num. Meth. Fluids 4, $43-69$ (1984).

6. U. Ghia, K. N. Ghia and C. T. Shin, High-Re solutions for incompressible flow using the Navier-Stokes equations and a multigrid method. J. Comp. Phys. 48, 387-411 (1982).

7. B. F. Armaly, F. Durst, J. C. F. Pereira and B. Schonung, Experimental and theoretical investigation of backwardfacing step flow. J. Fluid. Mech. 127, 473-496 (1983).

8. R. L. Lee, P. M. Gresho and R. L. Sani, Smoothing techniques for certain primitive variable solutions of the Navier-Stokes equations. Int. J. Num. Meth. Engng. 14, 1785-1804 (1979).

9. T. J. R. Hughes, K. Liu and A. Brooks, Finite element analysis of incompressible viscous flows by the penalty function formulation. J. Comput. Phys. 30, 1-60 (1979).

10. M. Bercovier and M. Engelman A finite element for the numerical solution of viscous incompressible flows. $J$. Comput. Phys. 30, 181-201 (1979).

11. S. C. R. Dennis and J. D. A. Walker, Calculation of the steady flow past a sphere at low and moderate Reynolds number. J. Fluid. Mech. 48, 771-789 (1971).

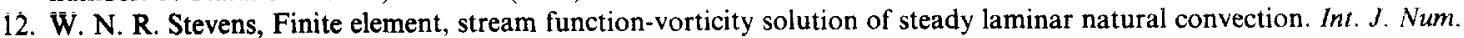
Meth. Fluids 2, 349-366 (1982).

13. J. W. Laminar, free convection in a vertical slot. J. Fluid. Mech. 23, 77-96 (1965).

14. G. K. Batchelor, Heat transfer by free convection across a closed-cavity between vertical boundaries at different temperature. Q. J. appl. Math. 209-233 (1954).

15. G. De Vahl Davis, Laminar natural convection in an enclosed rectangular cavity. Int. J. Heat Mass Transfer 11, 1675-1693 (1968).

16. A. Rubel and F. Landis, Numerical study of natural convection in a vertical rectangular enclosure. Phys. Fluids 12, 208-213 (1969).

17. J. D. Hellums and S. W. Churchill, Transient and steady state, free and natural convection, numerical solution. A.I.Ch.E.J. 8, 690-695 (1962).

18. J. Szekely and M. R. Todd, Natural convection in a rectangular cavity transient behavior and two phase system in laminar flow. Int. J. Heat Mass Transfer 14, 467-482 (1971).

19. R. J. Schnipke and J. G. Rice, A finite element method for free and forced convection heat transfer. Int. J. Num. Meth. Engng 24, 117-128 (1987). 